\title{
Wave Packet Dynamics for a Non-linear Schrödinger Equation Describing Continuous Position Measurements
}

\author{
C. Zander ${ }^{1}$, A.R. Plastino*2 and J. Díaz-Alonso ${ }^{3,4}$ \\ ${ }^{1}$ Physics Department, University of Pretoria, Pretoria 0002, South Africa \\ ${ }^{2}$ CeBio y Secretaria de Investigaciones, Universidad Nacional del Noroeste de la Prov. \\ de Buenos Aires-UNNOBA y CONICET, Roque Saenz Peña 456, Junín, Argentina \\ ${ }^{3}$ LUTH, Observatoire de Paris, CNRS, Universite Paris Diderot, 5 Place Jules Janssen, 92190 Meudon, France \\ ${ }^{4}$ Departamento de Física, Universidad de Oviedo, Calvo Sotelo 3300\%, Oviedo, Spain
}

(Dated: April 15, 2015)

\begin{abstract}
We investigate time-dependent solutions for a non-linear Schrödinger equation recently proposed by Nassar and Miret-Artés (NM) to describe the continuous measurement of the position of a quantum particle $[1,2]$. Here we extend these previous studies in two different directions. On the one hand, we incorporate a potential energy term in the NM equation and explore the corresponding wave packet dynamics, while in the previous works the analysis was restricted to the free-particle case. On the other hand, we investigate time-dependent solutions while previous studies focused on a stationary one. We obtain exact wave packet solutions for linear and quadratic potentials, and approximate solutions for the Morse potential. The free-particle case is also revisited from a time-dependent point of view. Our analysis of time-dependent solutions allows us to determine the stability properties of the stationary solution considered in $[1,2]$. On the basis of these results we reconsider the Bohmian approach to the NM equation, taking into account the fact that the evolution equation for the probability density $\rho=|\psi|^{2}$ is not a continuity equation. We show that the effect of the source term appearing in the evolution equation for $\rho$ has to be explicitly taken into account when interpreting the NM equation from a Bohmian point of view.
\end{abstract}

\section{INTRODUCTION}

Non-linear Schrödinger equations provide useful tools for modelling diverse physical phenomena and constitute an interesting arena for the analysis of fundamental issues in physics [1-7]. They are also related to other non-linear equations of mathematical physics, such as the non-linear Fokker-Planck equations [8-11]. Indeed, research on non-linear Schrödinger equations constitutes an important component of the more general line of enquiry concerning the exploration of non-linear extensions or modifications of some of the equations governing fundamental laws of physics (see for instance [12-14] and references therein). A non-linear Schrödinger equation was recently proposed by Nassar and Miret-Artés (NM) as an effective description of the evolution of a quantum particle subjected to a continuous position measurement process $[1,2]$.

The measurement of the position of quantum particles plays an essential role among the set of all possible physical measurements. Actually most physical measurements, if not even all of them, can be reduced to the measurement of the position of particles $[15,16]$. It is due to this that position observables are of fundamental importance in numerous approaches to the quantum measurement problem and related aspects of the foundations of quantum mechanics. Among the interesting proposals that focus on particles' position and which deal with fundamental aspects of quantum mechanics, are the de Broglie-Bohm pilot wave approach to quantum mechanics [17-19], the aforementioned non-linear extension of the Schrödinger equation describing the continuous measurement of the position of a particle [1, 2], the Ghirardi-Rimini-Weber model of wave function collapse [20], the entropic-dynamics approach to quantum evolution [21], and the Fisher information-based derivation of the fundamental Lagrangians which leads to relativistic wave equations [22]. Last but not least, an intriguing new perspective on the preponderance of the position observable in our perception of Nature has been recently advanced by Tegmark [23], suggesting possible connections between this issue and the physical basis of consciousness.

In the present contribution we revisit the abovementioned NM approach to continuous position measurement processes, investigating time-dependent solutions of the associated non-linear Schrödinger equation. NM considered the measurement of the position of a free particle, that is, "free" except from the effects arising from the measurement itself, which are modelled by a non-linear term in the Schrödinger equation $[1,2]$. Here we consider the measurement of the position of a particle moving under the effect of a potential $V(x)$. Our present study goes beyond previous works

*arplastino@unnoba.edu.ar 
also because we consider explicitly time-dependent solutions to the NM equation, while previous studies focused on a stationary solution. We obtain exact solutions for linear and quadratic potentials, and also develop a time-dependent wave packet approximation which we illustrate for the Morse potential. These time-dependent solutions allow us to determine the stability properties of the previously studied stationary solutions. On the basis of these developments we then reconsider the Bohm approach to the NM treatment of the position measurement process.

\section{PRELIMINARIES}

\section{A. Bohm's pilot wave model of quantum mechanics}

We now briefly review the Bohm approach to quantum mechanics, which will play a central role in our later considerations.

The de Broglie - Bohm theory, also called the pilot-wave theory or the quantum theory of motion, is an interpretation of quantum theory which has attracted the attention of researchers in recent years $[1,2,15,17-19,24-37]$. In their review of 100 years of quantum physics when celebrating the first hundred years of quantum physics, Tegmark and Wheeler considered Bohmian mechanics one of the most significant elements in its history [27]. Among the fascinating facets of Bohm's theory one can mention its relevance for the study of quantum chaos [32] and for the quantum measurement problem [1, 2, 19], and its applications to quantum cosmology [28-31]. Thermal equilibrium of quantum systems [34], entanglement [33], and the dynamics of quantum particles with position-dependent effective mass [35] have also been recently considered within Bohm's approach. An intriguing extension of quantum mechanics proposed by Valentini which has as its basis Bohmian dynamics, leads to specific astrophysical and cosmological predictions that might be observationally tested [36, 37].

In Bohm's model the particles constituting the system are assumed to have well defined positions in configuration space, in addition to involving a wave function. The temporal evolution of the particles' configuration is governed by the wave function via a "guiding" equation, but the time-evolution of the wave function is determined by the Schrödinger equation. The probability density in configuration space, given by the squared modulus of the wave function, contains our only knowledge about the positions of the system's particles. Bohm's approach yields a possible solution for the measurement problem, since the outcome of an experiment is registered by the configuration of the particles of the experimental apparatus after the completion of the experiment. The Bohmian model thus emphasizes an essential aspect of the measurement process, which is that "... all experiments and certainly all measurements in physics are in the last analysis essentially kinematic, for they are ultimately based on observations of the position of a particle or of a pointer on a scale as a function of time" [16]. The Bohmian description of the measurement process is fully compatible with Born's rule in standard quantum mechanics and the experimental predictions of Bohm's theory are identical to those of the usual quantum mechanical formalism (see, however, Valentini's Bohm-based proposal for an extension of quantum theory [36, 37]). The basic quantal non-locality is explicitly expressed in the de Broglie - Bohm formalism. In fact, the Bell inequalities were inspired by Bell's reaction to the work of Bohm [15].

It must be emphasized that the Bohmian dynamics incorporates the whole Hilbert space formalism of the standard formulation of quantum mechanics, for instance, a quantum particle has an associated wave function $\psi(\mathbf{r}, t)$ which obeys the Schrödinger equation of motion. The novel element of the Bohmian approach is that a quantum particle is now also endowed with a definite position $\mathbf{r}$ that evolves in time, as in classical mechanics, according to the equation $d \mathbf{r} / d t=\mathbf{p} / m=\mathbf{v}$, where $\mathbf{p}$ is the particle's linear momentum and $\mathbf{v}$ is the velocity of the particle. The particle's position $\mathbf{r}$ is an example of a hidden variable. The result of a position measurement is not predictable, however, it is regarded as predetermined. Actually, according to the Bohm approach, this is what happens with any kind of measurement as all measurements at some stage manifest themselves in the position of particles [15].

The Bohmian dynamics differs from standard classical dynamics in a fundamental manner, since the linear momentum $\mathbf{p}$ and the velocity $\mathbf{v}$ are not free variables anymore. They are, instead, determined by the particle's wave function $\psi$ and position $\mathbf{r}$. That is, the particle's motion is governed by a velocity field $\mathbf{v}(\mathbf{r}, t)$ which is, in turn, determined by a time-dependent solution of Schrödinger's equation $\psi(\mathbf{r}, t)$ by means of

$$
\mathbf{v}(\mathbf{r}, t)=-\frac{i \hbar}{2 m}\left[\psi^{-1}(\mathbf{r}, t) \nabla(\psi(\mathbf{r}, t))-\psi^{*-1}(\mathbf{r}, t) \nabla\left(\psi^{*}(\mathbf{r}, t)\right)\right] .
$$

In Bohm's theory a quantum particle has a definite position $\mathbf{r}$ whose temporal evolution is governed by the evolution 
equation

$$
\frac{d \mathbf{r}}{d t}=\mathbf{v}(\mathbf{r}, t)
$$

where the velocity field $\mathbf{v}(\mathbf{r}, t)$ is given by (1). When one prepares a state of the particle, however, one has neither control over the particular value adopted by $\mathbf{r}$ nor knowledge thereof. What one does know is that the probability density $\rho(\mathbf{r}, t)$ associated with the different possible particle's positions is given by

$$
\rho(\mathbf{r}, t)=|\psi(\mathbf{r}, t)|^{2}
$$

The velocity field (2) for the particle leads to the Liouville-like continuity equation for the probability density $\rho$, namely

$$
\frac{\partial \rho}{\partial t}+\nabla \cdot(\mathbf{v} \rho)=0
$$

It follows from the form (1) of the velocity field, the Schrödinger equation and the continuity equation (4), that if at the starting time $t=0$ the ensemble of initial positions for the particle is given by equation (3), then at all times $t$ the (time-dependent) density is also given by (3).

\section{B. The NM equation with a potential energy term}

The non-linear Schrödinger equation (NM equation) proposed by Nassar in [1] and extended in [2] to include a dissipative term, reads

$$
i \hbar \frac{\partial \psi(x, t)}{\partial t}=\left[H(x, t)+i \hbar\left(W_{c}(x, t)+W_{f}(x, t)\right)\right] \psi(x, t)
$$

where $H=-\frac{\hbar^{2}}{2 m} \frac{\partial^{2}}{\partial x^{2}}+V(x)$ is the standard Hamiltonian for a particle of mass $m$ under the potential $V(x)$,

$$
\begin{gathered}
W_{c}(x, t)=-\kappa\left[\ln |\psi(x, t)|^{2}-\left\langle\ln |\psi(x, t)|^{2}\right\rangle\right], \\
W_{f}(x, t)=-\frac{\nu}{2}\left[\ln \frac{\psi(x, t)}{\psi^{*}(x, t)}-\left\langle\ln \frac{\psi(x, t)}{\psi^{*}(x, t)}\right\rangle\right], \\
\left\langle\ln |\psi(x, t)|^{2}\right\rangle=\int|\psi(x, t)|^{2} \ln |\psi(x, t)|^{2} d x,
\end{gathered}
$$

$\kappa$ is a constant related to the resolution of position measurement and $\nu$ is a friction coefficient. The non-linear logarithmic term $W_{c}$ introduced by Nassar in [1] was inspired by Mensky's path integral approach to continuous measurements [3], whereas the term $W_{f}$ introduced in [2] was motivated by Kostin's work in connection with quantum dissipative systems [4]. In $[1,2]$ only the free-particle case $(V(x)=0)$ was considered.

In order to formulate the Bohmian dynamics associated with the NM equation, it is convenient to express the wave function explicitly in terms of its modulus and phase,

$$
\psi(x, t)=\phi(x, t) \exp [i S(x, t) / \hbar] .
$$

Then the probability density of the Bohmian particles is

$$
\rho(x, t)=|\phi(x, t)|^{2}
$$

and the Bohmian velocity field can be cast as

$$
u(x, t)=\frac{1}{m} \frac{\partial S(x, t)}{\partial x} .
$$


It is also useful to introduce the "quantum potential"

$$
V_{q}(x, t)=-\frac{\hbar^{2}}{2 m \phi(x, t)} \frac{\partial^{2} \phi(x, t)}{\partial x^{2}}
$$

Inserting $\psi=\phi \exp [i S / \hbar]$ into $(5)$ and taking into account the definitions of $u$ and $V_{q}$, one obtains the following differential equations for $\rho(x, t)$ and $u(x, t)$,

$$
\begin{gathered}
\frac{\partial \rho}{\partial t}=-\frac{\partial}{\partial x}(\rho u)-2 \kappa[\ln \rho-\langle\ln \rho\rangle] \rho \\
\frac{\partial u}{\partial t}+u \frac{\partial u}{\partial x}=-\frac{1}{m} \frac{\partial}{\partial x}\left[V_{q}+V\right]-\nu u .
\end{gathered}
$$

The friction term $W_{f}$ in the non-linear Schrödinger equation (5) leads to the term $-\nu u$ in the right hand side of the differential equation (14) governing the velocity field, which can be regarded as an extra drag force besides the force due to the potential $V(x)$ and the "quantum potential" $V_{q}$.

\section{WAVE PACKET DYNAMICS}

\section{A. Gaussian ansatz and its evolution}

Now we are going to investigate time-dependent solutions to the equations (13) and (14) governing respectively the probability density and the Bohmian velocity field associated with wave packet solutions of the NM non-linear Schrödinger equation. We start with a time-dependent Gaussian ansatz for the probability density,

$$
\rho(x, t)=|\psi(x, t)|^{2}=\left[2 \pi \delta^{2}(t)\right]^{-\frac{1}{2}} \exp \left[-\frac{(x-\bar{x}(t))^{2}}{2 \delta^{2}(t)}\right],
$$

where $\bar{x}(t)=\int x|\psi(x, t)|^{2} d x$ is the location of the wave packet's center (later to be identified with the classical trajectory of the particle) and $\delta(t)$ is the dispersion of the wave packet. Computing directly from (15) the expressions for $\frac{\partial \rho}{\partial t}$ and $2 \kappa[\ln \rho-\langle\ln \rho\rangle] \rho$, and inserting them in (13), one can verify that the velocity field $u(x, t)$ has to be given by

$$
u(x, t)=\left(\frac{\dot{\delta}}{\delta}-\kappa\right)(x-\bar{x})+\dot{\bar{x}} .
$$

Using now the ansatz (15) and equation (16), one can rewrite (14) as

$$
\left[\ddot{\delta}+(\nu-2 \kappa) \dot{\delta}+\left(\kappa^{2}-\kappa \nu\right) \delta-\frac{\hbar^{2}}{4 m^{2} \delta^{3}}\right](x-\bar{x})+\delta(\ddot{\bar{x}}+\nu \dot{\bar{x}})=-\frac{\delta}{m} \frac{\partial V}{\partial x} .
$$

Notice that, in order for (14) to hold, equation (17) must be satisfied for all $x$ and all $t$. In equation (17), $\delta(t), \dot{\delta}(t), \ddot{\delta}(t), \bar{x}(t), \dot{\bar{x}}(t), \ddot{\bar{x}}(t)$ are functions of $t$, and $\frac{\partial V}{\partial x}$ is a function of $x$.

Now we shall use the "wave packet approximation" [38]. The main assumption of the approximation is that, within the spatial range where the wave function is appreciably different from zero, the potential $V(x)$ changes slowly enough so that it can be approximated to second-order in $(x-\bar{x})$

$$
V(x) \approx V(\bar{x})+\left.(x-\bar{x}) \frac{\partial V}{\partial x}\right|_{x=\bar{x}}+\left.\frac{1}{2}(x-\bar{x})^{2} \frac{\partial^{2} V}{\partial x^{2}}\right|_{x=\bar{x}} .
$$

Thus

$$
\left.\frac{\partial V}{\partial x} \approx \frac{\partial V}{\partial x}\right|_{x=\bar{x}}+\left.(x-\bar{x}) \frac{\partial^{2} V}{\partial x^{2}}\right|_{x=\bar{x}}
$$


and so combining (17) and (19) we obtain

$$
\begin{aligned}
\left(\ddot{\delta}+(\nu-2 \kappa) \dot{\delta}+\left(\kappa^{2}-\kappa \nu\right) \delta\right. & \left.-\frac{\hbar^{2}}{4 m^{2} \delta^{3}}+\left.\frac{\delta}{m} \frac{\partial^{2} V}{\partial x^{2}}\right|_{x=\bar{x}}\right)(x-\bar{x}) \\
& +\delta\left(\ddot{\bar{x}}+\nu \dot{\bar{x}}+\left.\frac{1}{m} \frac{\partial V}{\partial x}\right|_{x=\bar{x}}\right)=0
\end{aligned}
$$

Note that in (20), the quantities $\left.\frac{\partial V}{\partial x}\right|_{x=\bar{x}(t)}$ and $\left.\frac{\partial^{2} V}{\partial x^{2}}\right|_{x=\bar{x}(t)}$ are evaluated at the (time dependent) center of the wave packet $\bar{x}(t)$. These quantities are therefore functions only of the time $t$ (through $\bar{x}(t)$ ). Now, since $(20)$ must hold for all $x$ and $t$, we have

$$
\begin{gathered}
\ddot{\delta}+(\nu-2 \kappa) \dot{\delta}+\left(\kappa^{2}-\kappa \nu\right) \delta-\frac{\hbar^{2}}{4 m^{2} \delta^{3}}+\left.\frac{\delta}{m} \frac{\partial^{2} V}{\partial x^{2}}\right|_{x=\bar{x}}=0 \\
\ddot{\bar{x}}+\nu \dot{\bar{x}}+\left.\frac{1}{m} \frac{\partial V}{\partial x}\right|_{x=\bar{x}}=0 .
\end{gathered}
$$

Notice that the approximation implied by equations (18) and (19) becomes exact in the case of constant, linear or quadratic potentials. In those cases, therefore, the Gaussian ansatz yields an exact solution, described by equations (21) and (22), that govern the evolution of the centre $\bar{x}(t)$ and the width of the Gaussian wave packet $\delta(t)$. Otherwise, the Gaussian solution is only approximate. Notice that equation (21) can be cast under the guise,

$$
\ddot{\delta}=(2 \kappa-\nu) \dot{\delta}-\frac{\partial W}{\partial \delta},
$$

which can formally be regarded as the equation of motion of a particle of unit mass moving in one dimension with coordinate $\delta$ under the effect of a drag force given by $(2 \kappa-\nu) \dot{\delta}$ and an effective potential $W$ given by

$$
W(\delta, t)=\frac{1}{2}\left(\kappa^{2}-\kappa \nu\right) \delta^{2}+\frac{\hbar^{2}}{8 m^{2} \delta^{2}}+\left.\frac{1}{2 m} \delta^{2} \frac{\partial^{2} V}{\partial x^{2}}\right|_{x=\bar{x}}
$$

Equation (22) says that $\bar{x}(t)$ describes a classical, Newtonian particle moving in the potential $V(x)$ and under the effect of a drag force $\nu \dot{\bar{x}}$.

The evolution equations (21) and (22) for the dispersion $\delta$ and centre $\bar{x}$ of the Gaussian density (15) can also be derived by directly considering a Gaussian ansatz for the wave function $\psi(x, t)$ evolving according to the NM Schrödinger equation. In this alternative procedure one deals with the NM equation without recourse to the Bohmian approach. Let us then consider a Gaussian wave function

$$
\psi(x, t)=e^{a(t) x^{2}+b(t) x+c(t)}
$$

where $a, b$ and $c$ are complex functions of time, i.e. $a(t)=a_{R}(t)+i a_{I}(t)$ and similarly for $b$ and $c$. Substituting (25) into the non-linear Schrödinger equation (5), one finds that the ansatz (25) constitutes an exact solution of (5) provided that the complex parameters $a, b$ and $c$ satisfy the set of ordinary differential equations,

$$
\begin{aligned}
\dot{a}= & \frac{2 i \hbar}{m} a^{2}-2 \kappa a_{R}-i \nu a_{I}-\left.\frac{i}{2 \hbar} \frac{\partial^{2} V}{\partial x^{2}}\right|_{x=\bar{x}} \\
\dot{b}= & \frac{2 i \hbar}{m} a b-2 \kappa b_{R}-i \nu b_{I}-\frac{i}{\hbar}\left[\left.\frac{\partial V}{\partial x}\right|_{x=\bar{x}}-\left.\bar{x} \frac{\partial^{2} V}{\partial x^{2}}\right|_{x=\bar{x}}\right] \\
\dot{c}= & \frac{i \hbar}{2 m}\left(2 a+b^{2}\right)-\frac{i}{\hbar}\left[V(\bar{x})-\left.\bar{x} \frac{\partial V}{\partial x}\right|_{x=\bar{x}}+\left.\frac{1}{2} \bar{x}^{2} \frac{\partial^{2} V}{\partial x^{2}}\right|_{x=\bar{x}}\right] \\
& -2 \kappa\left[c_{R}+\sqrt{\frac{\pi}{-2 a_{R}}} e^{2 c_{R}-\frac{b_{R}^{2}}{2 a_{R}}}\left\{\frac{1}{4}-c_{R}+\frac{b_{R}^{2}}{4 a_{R}}\right\}\right] \\
& -i \nu\left[c_{I}+\sqrt{\frac{\pi}{-2 a_{R}}} e^{2 c_{R}-\frac{b_{R}^{2}}{2 a_{R}}}\left\{\frac{a_{I}}{4 a_{R}}-c_{I}+\frac{b_{R} b_{I}}{2 a_{R}}-a_{I}\left(\frac{b_{R}}{2 a_{R}}\right)^{2}\right\}\right]
\end{aligned}
$$


which are equivalent to six first-order coupled differential equations for the real and imaginary parts of $a, b$ and $c$. The two equations contained in (26), governing the evolution of $a_{R}$ and $a_{I}$, do not involve the coefficients $b$ or $c$. Indeed, we have,

$$
\begin{aligned}
\dot{a}_{R} & =-\frac{4 \hbar}{m} a_{R} a_{I}-2 \kappa a_{R} \\
\dot{a}_{I} & =\frac{2 \hbar}{m}\left(a_{R}^{2}-a_{I}^{2}\right)-\nu a_{I}-\left.\frac{1}{2 \hbar} \frac{\partial^{2} V}{\partial x^{2}}\right|_{x=\bar{x}} .
\end{aligned}
$$

The two equations in (29) are equivalent to a second-order differential equation for $a_{R}$. Indeed, it can be verified after some algebra that (29) is equivalent to

$$
\ddot{a}_{R}-\frac{3}{2} \frac{\dot{a}_{R}^{2}}{a_{R}}+(\nu-2 \kappa) \dot{a}_{R}-2\left(\kappa^{2}-\kappa \nu\right) a_{R}+\frac{8 \hbar^{2}}{m^{2}} a_{R}^{3}-\left.\frac{2}{m} \frac{\partial^{2} V}{\partial x^{2}}\right|_{x=\bar{x}} a_{R}=0 .
$$

When dealing with equation (30), the initial conditions are given by $a_{R}(0)$ and $\dot{a}_{R}(0)$, instead of $a_{R}(0)$ and $a_{I}(0)$. Equating the modulus squared of equation (25) with (15), we obtain a relation between $a_{R}$ and $\delta$, namely

$$
a_{R}=-\frac{1}{4 \delta^{2}}
$$

Using this we recover equation (21) from (30). The above makes it clear why the differential equation (21) is secondorder in time despite the non-linear Schrödinger equation being first-order in time.

\section{B. Stationary solutions and their stability}

In $[1,2]$ attention was focused on a solution of the NM non-linear Schrödinger equation that is stationary in the sense of having $\dot{\delta}=\ddot{\delta}=0$. That is, this solution describes a wave packet of constant width. Here we are going to analyze the stability of this solution. The stability depends on the relationship between $\nu, \kappa$ and $\left.\frac{\partial^{2} V}{\partial x^{2}}\right|_{x=\bar{x}}$, as will become clear from the following discussion. For stationary solutions $\left(\dot{\delta}=\ddot{\delta}=0 ; \delta=\delta_{s}\right)$ to be stable we can see from (23) that we necessarily require that $\kappa<\frac{\nu}{2}$, otherwise the overall effect of the drag term in the right hand side of (23) will not be dissipative and the width of the wave packet would diverge. We are only going to analyse the stability criterion when $\left.\frac{\partial^{2} V}{\partial x^{2}}\right|_{x=\bar{x}}=V^{\prime \prime}$, where $V^{\prime \prime}$ is a constant and thus the wave packet approximation is exact. Then the "effective potential" $W$ given in (24) only depends on $\delta$ (that is, $W$ is time-independent). The additional requirement for stability is now that $W$ has to have a local minimum at the stationary solution $\delta=\delta_{s}$. In other words, we must have $W^{\prime}\left(\delta_{s}\right)=0$ and $W^{\prime \prime}\left(\delta_{s}\right)>0$. Under these conditions the "drag effect" ensures that the particle looses "energy" and thus reaches the potential minimum at $\delta_{s}$, where it will remain (here, when using the expressions "particle" and "energy" we refer to the fact that the equation of motion for $\delta$, as already said, looks like the Newtonian equation of motion of a particle in one dimension with coordinate $\delta$ ). Taking both requirements into account results in the following stability condition (see Appendix A for details),

$$
\begin{array}{llll}
\kappa<\frac{\nu}{2} & \text { if } & \frac{\nu^{2}}{4}-\frac{V^{\prime \prime}}{m}<0 \\
\kappa<\frac{\nu}{2}-\sqrt{\frac{\nu^{2}}{4}-\frac{V^{\prime \prime}}{m}} & \text { if } & \frac{\nu^{2}}{4}-\frac{V^{\prime \prime}}{m} \geq 0 .
\end{array}
$$

When these conditions are fulfilled the stable stationary value of $\delta$ is given by,

$$
\delta_{s}^{2}=\frac{\hbar^{2}}{4 m^{2}}\left(\kappa^{2}-\kappa \nu+\frac{V^{\prime \prime}}{m}\right)^{-1} .
$$

The stationary value $\delta_{s}$ can be interpreted as representing the resolution of the spatial measurement process. We see that for a free particle with $\nu=0$ this resolution is $\delta_{s}=\frac{\hbar}{2 m|\kappa|}$. That is, the measurement resolution is basically determined by the absolute value of the parameter $\kappa$.

The detailed analysis of the wave-packet dynamics for the linear and quadratic cases is given in Subsections III C and III D. The general case of the wave packet approximation where $\left.\frac{\partial^{2} V}{\partial x^{2}}\right|_{x=\bar{x}}$ is time-dependent, and thus $W$ as well, 
has to be treated individually for each potential.

When we have a stable stationary solution $\delta=\delta_{s}$, the characteristic time $\tau$ it takes for $\delta(t)$ to reach the stationary value $\delta_{s}$ can be estimated by considering the time change (due to the drag term in $(23)$ ) of the "energy" $\varepsilon(t)=$ $\frac{1}{2} \dot{\delta}(t)^{2}+W(\delta(t))$. An estimate of $\tau$ is then given by,

$$
\tau=\frac{\varepsilon_{s}-\varepsilon_{i}}{\dot{\varepsilon}(0)}
$$

where $\varepsilon_{i}$ and $\varepsilon_{s}$ are the initial and final (relaxed) energies respectively, and

$$
\begin{aligned}
\dot{\varepsilon} & =\dot{\delta}\left[\ddot{\delta}+\frac{d W}{d \delta}\right] \\
& =\dot{\delta}^{2}(2 \kappa-\nu) .
\end{aligned}
$$

To obtain the second equality in the above equation one substitutes $\ddot{\delta}$ by the expression on the right hand side of the equation of motion (23). To get an order of magnitude estimate for the relaxation time, we evaluate $\varepsilon_{i}$ at the initial conditions $\left(\delta(0)=\delta_{s} ; \dot{\delta}(0)=\dot{\delta}_{0}\right)$, and $\varepsilon_{s}$ at the final conditions $\left(\delta(0)=\delta_{s} ; \dot{\delta}(0)=0\right)$. The quantity $\dot{\varepsilon}(0)$ is also evaluated at the initial time, yielding $\dot{\varepsilon}(0)=\dot{\delta}_{0}^{2}(2 \kappa-\nu)$. This leads to the estimate,

$$
\tau=\frac{1}{2(\nu-2 \kappa)} .
$$

This is of the same order of magnitude as the Bohmian time constant in $[1,2]$ which gives the characteristic time it takes for the quantum trajectories to approach the classical one.

\section{Linear potential}

Let us first consider a linear potential $V(x)=V_{0}+V^{\prime} x$. In this case we have $\left.\frac{\partial^{2} V}{\partial x^{2}}\right|_{x=\bar{x}}=0$ and equations (21) and (22) become

$$
\begin{gathered}
\ddot{\delta}+(\nu-2 \kappa) \dot{\delta}+\left(\kappa^{2}-\kappa \nu\right) \delta-\frac{\hbar^{2}}{4 m^{2} \delta^{3}}=0 \\
\ddot{\bar{x}}=-\nu \dot{\bar{x}}-\frac{V^{\prime}}{m} .
\end{gathered}
$$

We rewrite equation (37) and give the solution to (38),

$$
\begin{gathered}
\ddot{\delta}=(2 \kappa-\nu) \dot{\delta}+\left(\nu \kappa-\kappa^{2}\right) \delta+\frac{\hbar^{2}}{4 m^{2} \delta^{3}} \\
\bar{x}(t)=\frac{1}{\nu}\left(1-e^{-\nu t}\right)\left(\dot{\bar{x}}(0)+\frac{V^{\prime}}{\nu m}\right)-\frac{V^{\prime}}{\nu m} t+x(0) .
\end{gathered}
$$

As already mentioned, equation (39) can be regarded as the equation of motion of a particle of unit mass, under the effect of a "potential"

$$
W(\delta)=\frac{1}{2}\left(\kappa^{2}-\nu \kappa\right) \delta^{2}+\frac{\hbar^{2}}{8 m^{2} \delta^{2}}
$$

and a "drag force" given by $(2 \kappa-\nu) \dot{\delta}$. The stability criterion $(32)$ ensures that the potential $W$ has a minimum, which means the real constant $\kappa$ has to be negative, $\kappa<0$. Due to the "drag force" the particle looses "energy" and tends to end up at $\delta_{s}$, the bottom of the potential well, given by $\left.\frac{d W}{d \delta}\right|_{\delta=\delta_{s}}=0$, yielding

$$
\delta_{s}=\frac{\hbar}{2 m \sqrt{\kappa^{2}-\nu \kappa}} .
$$


The above mentioned behavior can be verified by numerically solving equation (39).

For all the results depicted in the figures we have used units of mass, length and time such that $m=1$ and $\hbar=1$. Choosing $\nu=0,1$ and $\kappa=-1$, the plot for the potential $W$ is given in Figure 1. The numerical solution of equation (39) for $\nu=0,1, \kappa=-1$ and with different initial conditions is given in Figure 2. From that figure one can see that the "particle" indeed reaches a stationary state, which implies that the width of the wave packet then remains constant.

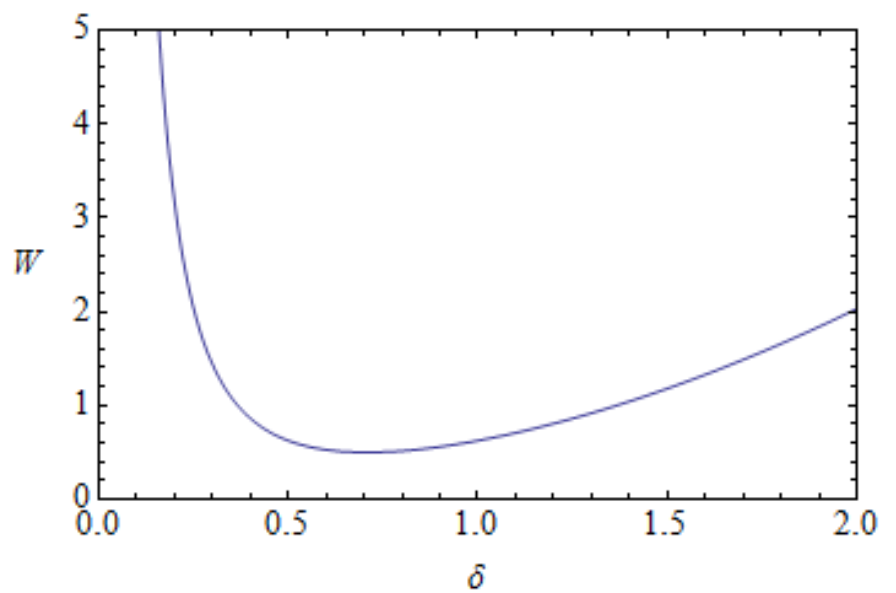

(a) $\nu=0$.

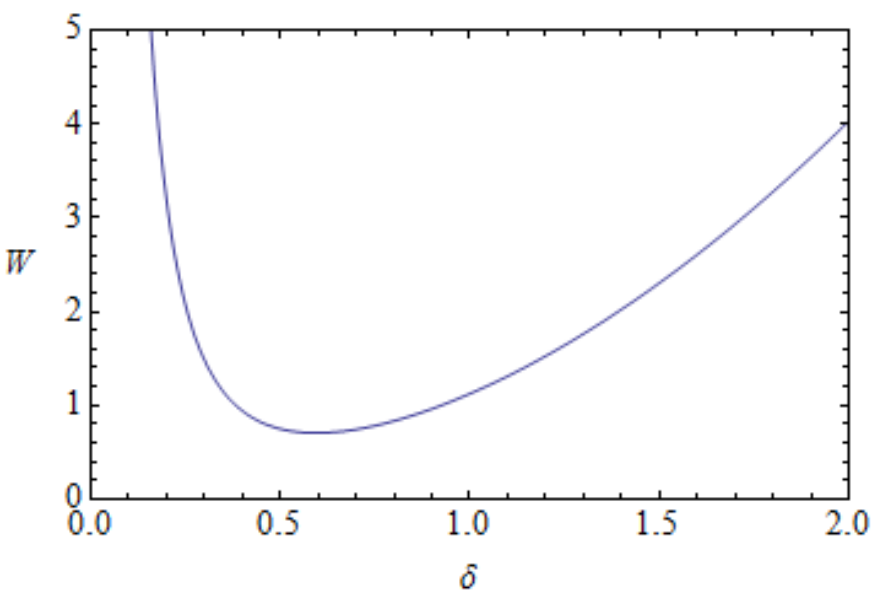

(b) $\nu=1$.

FIG. 1: Effective potential $W(41)$ governing the evolution of the width of the wave packet in a linear potential, as a function of the wave packet width $\delta$, for $\kappa=-1$ and different values for $\nu$.

\section{Quadratic potential}

We now consider a quadratic potential, $V(x)=V_{0}+V^{\prime} x+\frac{1}{2} V^{\prime \prime} x^{2}$ and therefore

$$
\begin{gathered}
\ddot{\delta}=(2 \kappa-\nu) \dot{\delta}+\left(\nu \kappa-\kappa^{2}\right) \delta+\frac{\hbar^{2}}{4 m^{2} \delta^{3}}-\frac{\delta V^{\prime \prime}}{m} \\
\ddot{\bar{x}}=-\nu \dot{\bar{x}}-\frac{V^{\prime}}{m}-\frac{V^{\prime \prime}}{m} \bar{x},
\end{gathered}
$$

where (44) is the equation of motion of a damped harmonic oscillator. Again, we can regard equation (43) as the equation of motion of a "particle" of unit mass, "coordinate" $\delta$, moving under the effect of a drag force $(2 \kappa-\nu) \dot{\delta}$ and a potential $W(\delta)$

$$
W(\delta)=\frac{1}{2}\left(\kappa^{2}-\kappa \nu\right) \delta^{2}+\frac{\hbar^{2}}{8 m^{2} \delta^{2}}+\frac{1}{2} \frac{V^{\prime \prime}}{m} \delta^{2}
$$

The minimum of this potential corresponds to the coordinate $\delta_{s}$ given by,

$$
\delta_{s}=\frac{\hbar}{2 m} \frac{1}{\sqrt{\kappa^{2}-\nu \kappa+\frac{V^{\prime \prime}}{m}}} .
$$

Again, due to the drag force, the "particle" moves towards the potential minimum at $\delta_{s}$ provided that condition (32) holds, which ensures a potential well and dissipative dynamics.

Choosing $\nu=0,1, \kappa=-1$ and $V^{\prime \prime}=1$, the plot for the potential $W$ is given in Figure 3 . The numerical solution of equation (43) for $\nu=0,1, \kappa=-1, V^{\prime \prime}=1$ and different initial conditions is given in Figure 4 . Once again the dispersion of the wave packet reaches a steady state. 


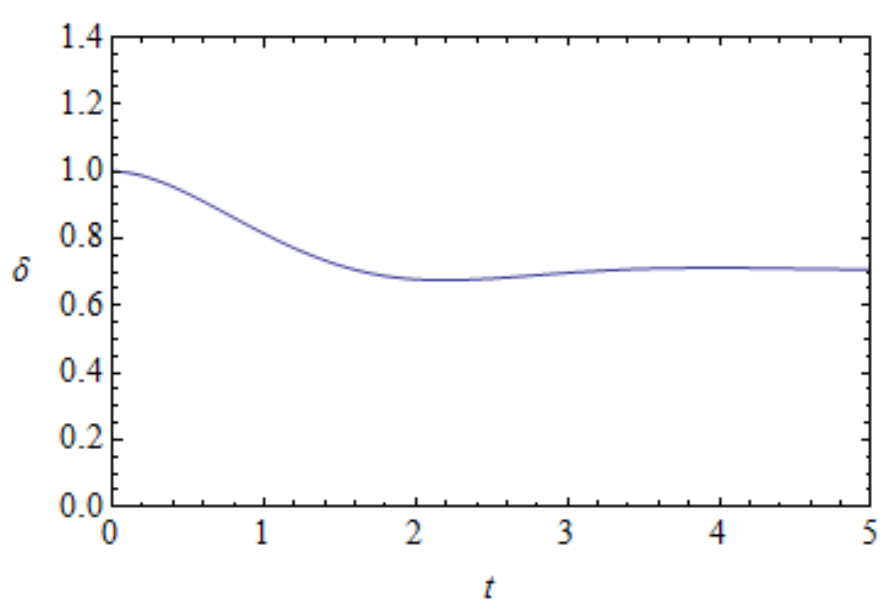

(a) $\nu=0$ and $\delta(0)=1, \delta^{\prime}(0)=0$.

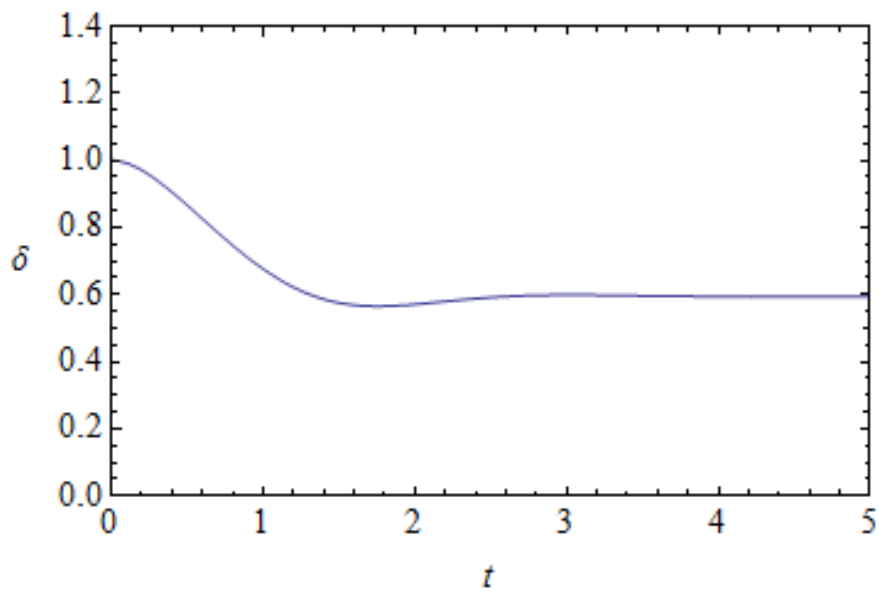

(c) $\nu=1$ and $\delta(0)=1, \delta^{\prime}(0)=0$.

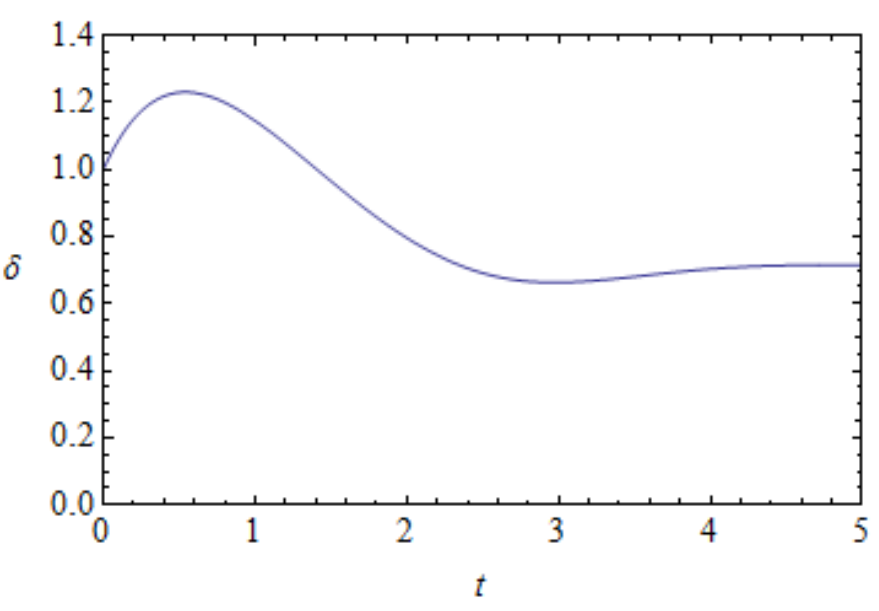

(b) $\nu=0$ and $\delta(0)=1, \delta^{\prime}(0)=1$.

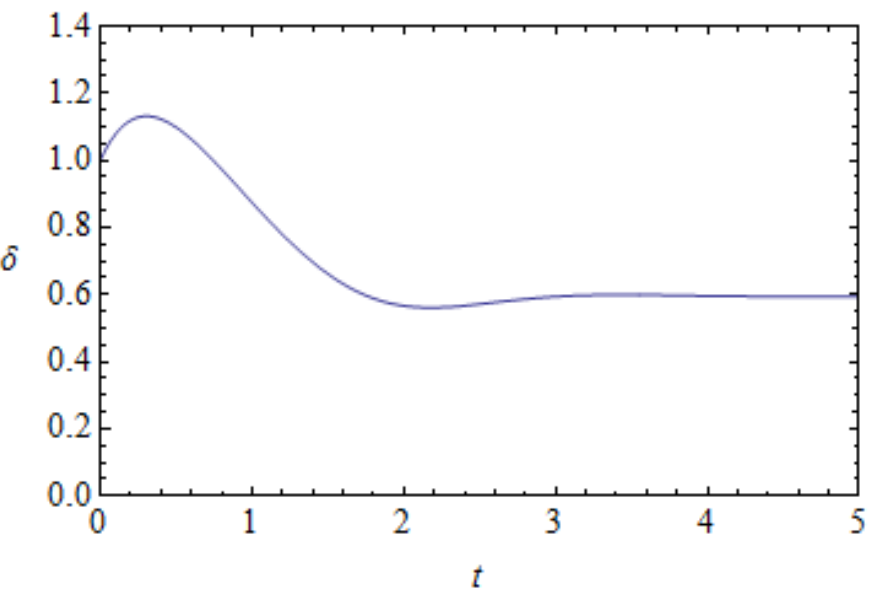

$(\mathrm{d}) \nu=1$ and $\delta(0)=1, \delta^{\prime}(0)=1$.

FIG. 2: Time evolution of the wave packet width, $\delta(t)$, in a linear potential for $\kappa=-1, \nu=0,1$, and different initial conditions $\delta(0)$ and $\delta^{\prime}(0)$.

\section{E. Morse potential}

The Morse potential is given by

$$
V(x)=-D+D\left[1-e^{-\alpha\left(x-x_{0}\right)}\right]^{2},
$$

where the minimum $-D$ occurs at point $x_{0}$. Using this potential, equation $(21)$ becomes $(\operatorname{setting} \nu=0)$

$$
\ddot{\delta}=2 \kappa \dot{\delta}-\kappa^{2} \delta+\frac{\hbar^{2}}{4 m^{2} \delta^{3}}-\frac{2 \alpha^{2} D}{m}\left(2 e^{-\alpha\left(\bar{x}-x_{0}\right)}-1\right) e^{-\alpha\left(x-x_{0}\right)} \delta
$$

and equation $(22)$ is then

$$
\ddot{\bar{x}}=-\left.\frac{1}{m} \frac{\partial V}{\partial x}\right|_{x=\bar{x}},
$$

which is the equation of motion of a Newtonian particle moving in the Morse potential. The equation of motion (49) admits exact analytical solutions [39]. These solutions can be conveniently expressed in terms of the dimensionless positive parameter $\mu=\frac{E+D}{D}$, where $E=\frac{1}{2} m \dot{\bar{x}}^{2}+V(\bar{x})$ is, of course, an integral of motion of equation (49). The 


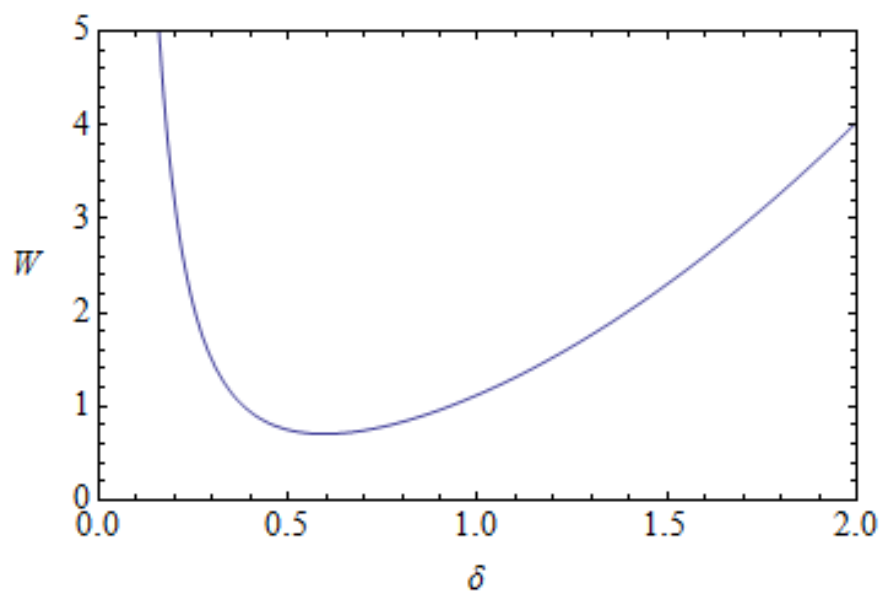

(a) $\nu=0$.

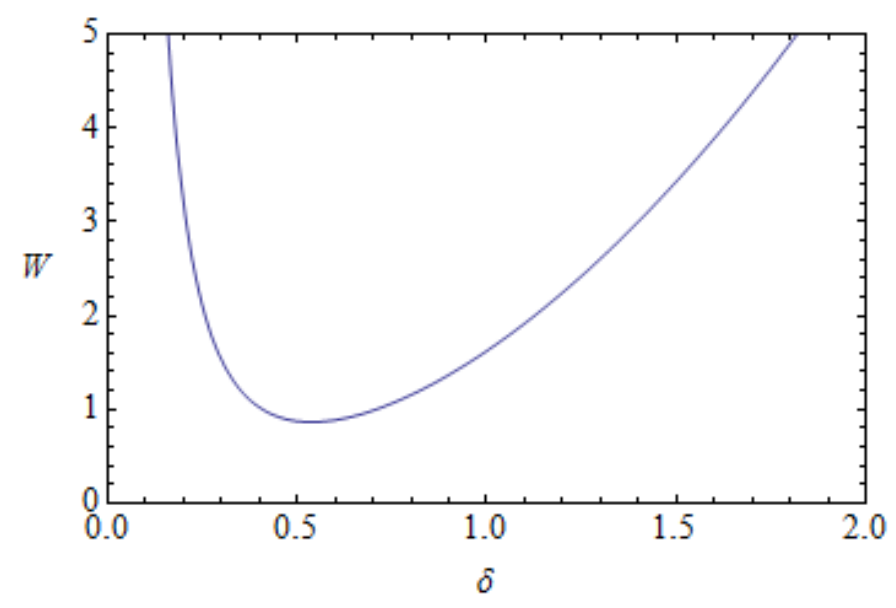

(b) $\nu=1$.

FIG. 3: Effective potential $W(45)$ governing the evolution of the width of the wave packet in a quadratic potential, as a function of the wave packet width $\delta$, for $\kappa=-1, V^{\prime \prime}=1$ and different values for $\nu$.

parameter $\mu$ determines whether the solution $\bar{x}(t)$ corresponds to oscillatory motion or not. For $\mu>1$ one has,

$$
\bar{x}(t)=x_{0}+\frac{1}{\alpha} \ln \left(\frac{\sqrt{\mu} \cosh \left[\gamma\left(t-t_{0}\right)\right]-1}{\mu-1}\right)
$$

with

$$
\gamma=\sqrt{\frac{2 \alpha^{2} D}{m}(\mu-1)},
$$

which gives unbound orbits, and for $0<\mu<1$

$$
\bar{x}(t)=x_{0}+\frac{1}{\alpha} \ln \left(\frac{1-\sqrt{\mu} \cos \left[\lambda\left(t-t_{0}\right)\right]}{1-\mu}\right)
$$

with

$$
\lambda=\sqrt{\frac{2 \alpha^{2} D}{m}(1-\mu)},
$$

the bound orbits are obtained. In both cases $\dot{\bar{x}}\left(t_{0}\right)=0$.

The numerical solution of equation (48) for either the unbound orbits (50) with $\mu=2$ or the bound orbits (52) with $\mu=0.5$ and for $\kappa=-1, D=1, \alpha=0.1, x_{0}=0, t_{0}=2$ and different initial conditions is given in Figure 5 . For the unbound orbits, the wave packet approximation becomes exact when the particle has escaped the potential, that is, when $\delta$ relaxes to a constant value given by

$$
\delta_{s}^{2}=\frac{\hbar}{2 m|\kappa|}
$$

As the magnitude of $\kappa$ increases, which corresponds to a greater non-linear effect, the dispersion of the wave packet decreases, which means the wave packet confinement is enhanced. When $\kappa \rightarrow 0$ we recover the wave packet behaviour for the linear Schrödinger equation. In the case of the bound orbits, $\delta$ approaches the same value as given in equation (54), however, it has periodic fluctuations when $\dot{\bar{x}}(t)=0$ at the left turning point in the potential. The amplitude of these fluctuations decreases when $\kappa$ becomes more negative. If $\kappa$ were to be positive, then the solution $\delta$ would diverge as shown in Figure 6 and the approximation becomes inaccurate. Our numerical results indicate that for $\kappa<-0.1$ the wave packet approximation is good and the solution $\delta(t)$ evolves towards a time-periodic attractor. 


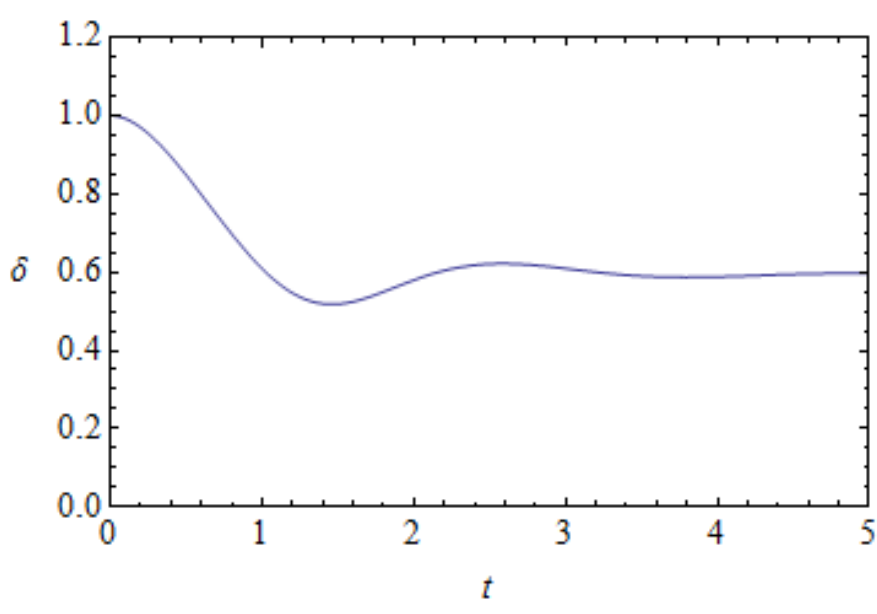

(a) $\nu=0$ and $\delta(0)=1, \delta^{\prime}(0)=0$.

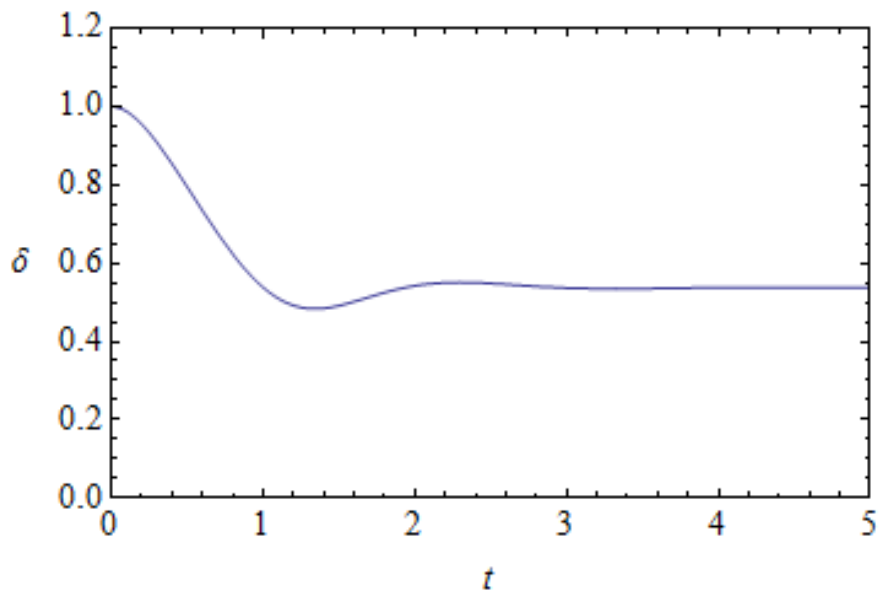

(c) $\nu=1$ and $\delta(0)=1, \delta^{\prime}(0)=0$.

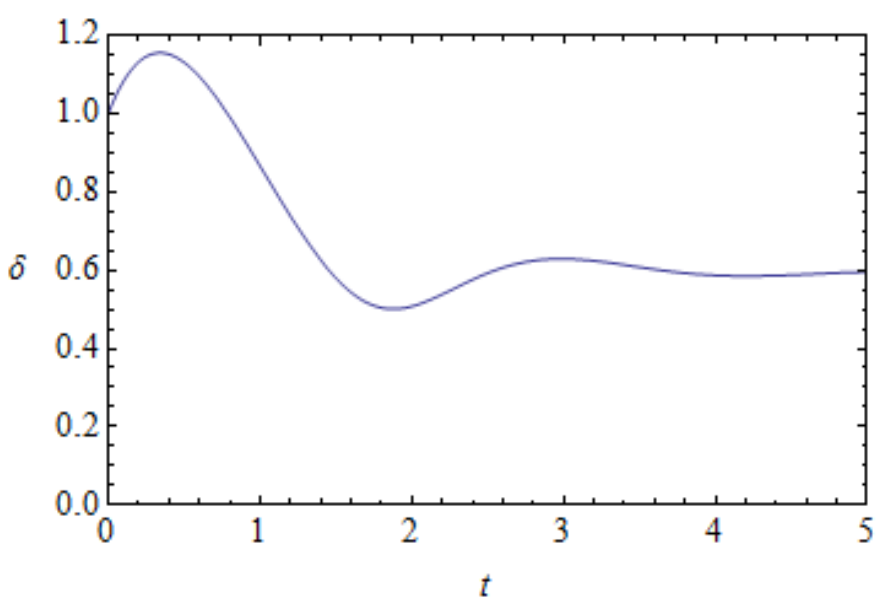

(b) $\nu=0$ and $\delta(0)=1, \delta^{\prime}(0)=1$.

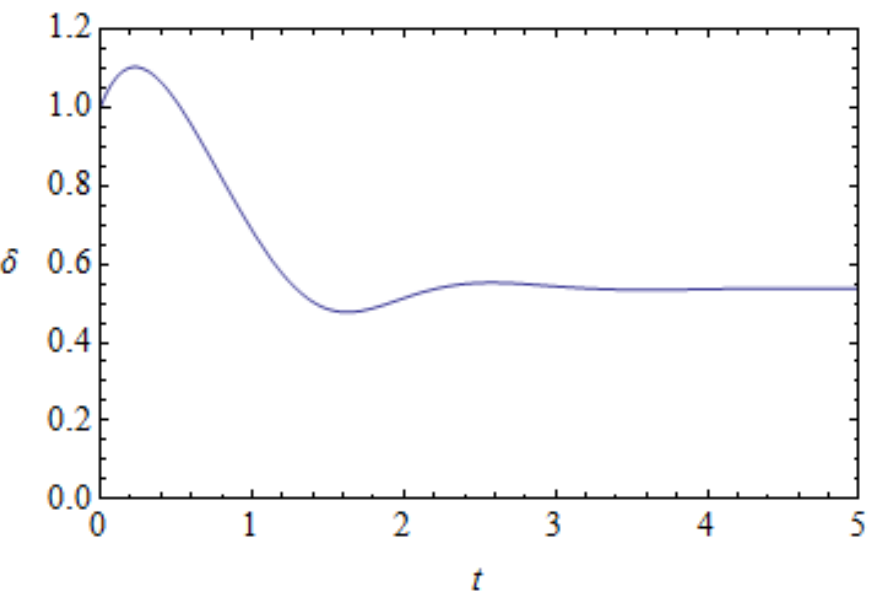

$(\mathrm{d}) \nu=1$ and $\delta(0)=1, \delta^{\prime}(0)=1$.

FIG. 4: Time evolution of the wave packet width, $\delta(t)$, in a quadratic potential for $\kappa=-1, \nu=0,1, V^{\prime \prime}=1$ and different initial conditions $\delta(0)$ and $\delta^{\prime}(0)$.

\section{THE BOHMIAN TRAJECTORIES}

In this section we are going to analyze the Bohmian trajectories corresponding to the time-dependent wave packet solutions of the NM evolution equation that we have considered in the previous sections. We are going to pay special attention to the relationship between the behavior of the Bohmian trajectories, on the one hand, and the stability properties of the wave packet solutions of constant width, on the other hand. The differential equation for the Bohmian trajectories is

$$
\frac{d x}{d t}=u(x, t)
$$

where $u(x, t)$ is given by equation (16). Consequently, it follows that

$$
\frac{d}{d t}(x-\bar{x})=\left(\frac{\dot{\delta}}{\delta}-\kappa\right)(x-\bar{x})
$$

and thus

$$
(x-\bar{x})=(x-\bar{x})_{0} \exp \left[\int_{0}^{t}\left(\frac{\dot{\delta}}{\delta}-\kappa\right) d t^{\prime}\right]
$$




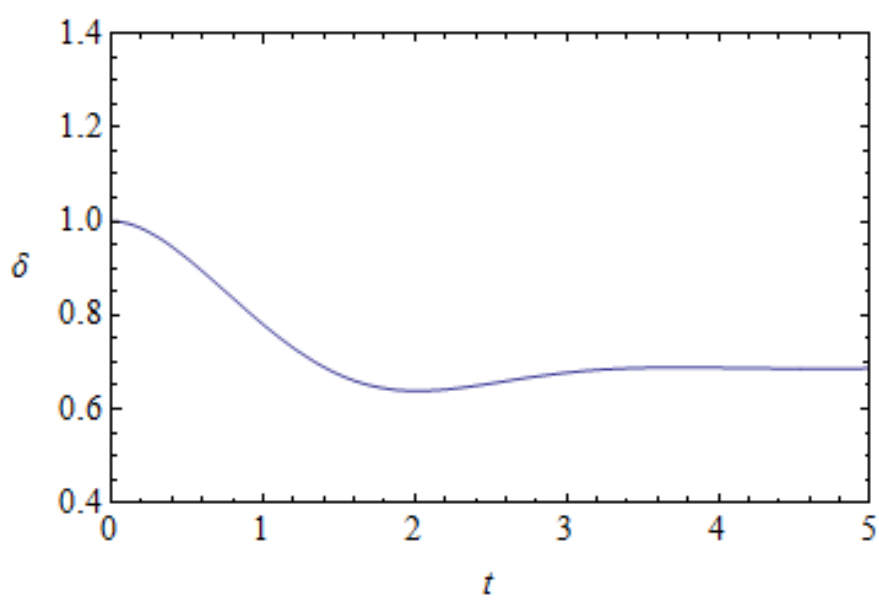

(a)Evolution of $\delta$ for an unbound classical orbit $\bar{x}(t)(50)$ for $\mu=2$ with $\delta(0)=1, \delta^{\prime}(0)=0$.

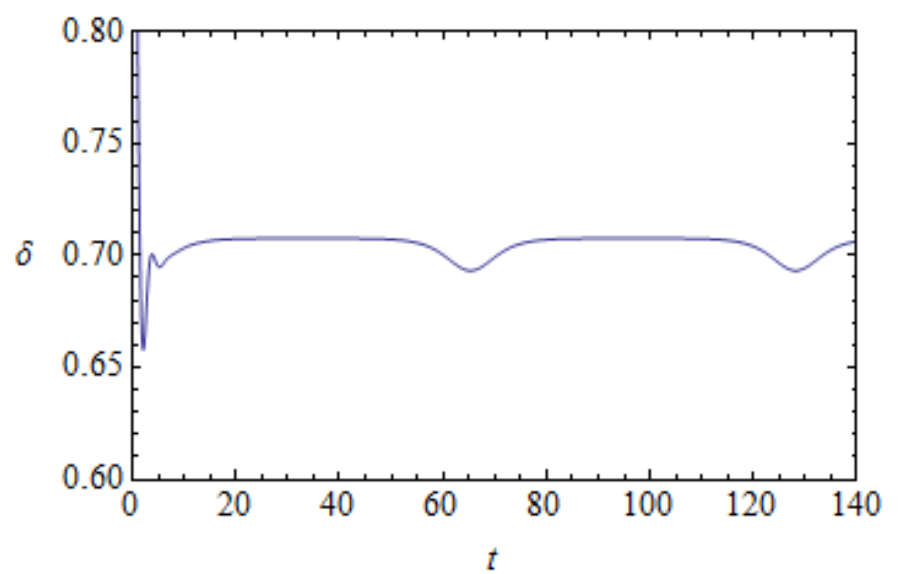

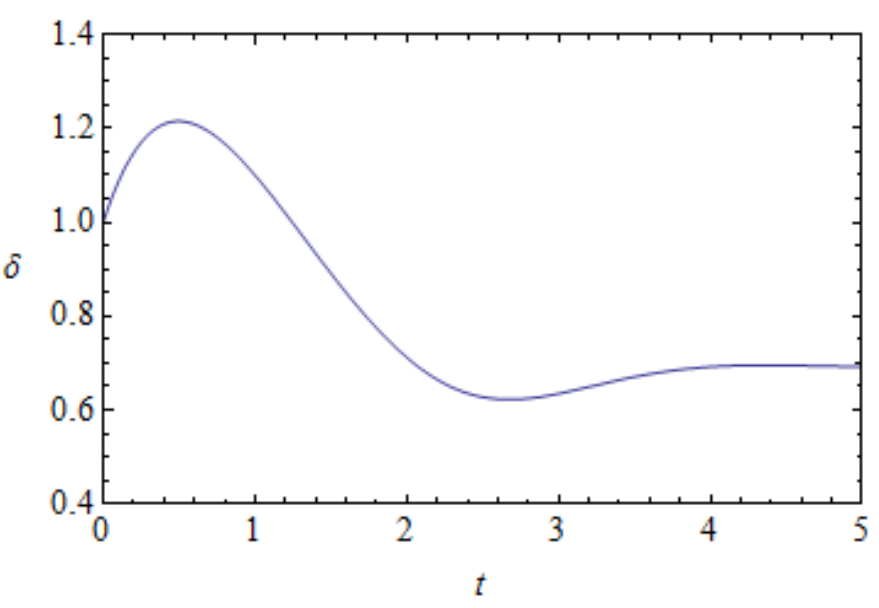

(b)Evolution of $\delta$ for an unbound classical orbit $\bar{x}(t)(50)$ for $\mu=2$ with $\delta(0)=1, \delta^{\prime}(0)=1$.

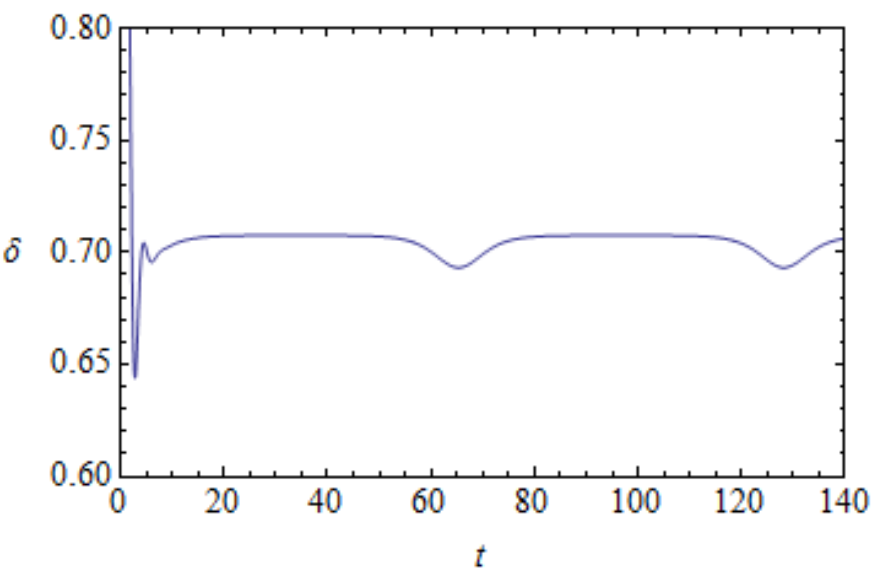

(c)Evolution of $\delta$ for a bound classical orbit $\bar{x}(t)(52)$ for $\mu=0.5$ with(d)Evolution of $\delta$ for a bound classical orbit $\bar{x}(t)(52)$ for $\mu=0.5$ with $\delta(0)=1, \delta^{\prime}(0)=0$ $\delta(0)=1, \delta^{\prime}(0)=1$.

FIG. 5: Time evolution of the wave packet width, $\delta(t)$, in a Morse potential for bound and unbound classical orbits $\bar{x}(t)$ of the wave packet center and different initial conditions $\delta(0)$ and $\delta^{\prime}(0)$. The relevant parameters are $\kappa=-1, D=1, \alpha=0.1, x_{0}=0$, $t_{0}=2$.

and so

$$
(x-\bar{x})=(x-\bar{x})_{0} \frac{\delta(t)}{\delta_{0}} e^{-\kappa t}
$$

where $\delta_{0}=\delta(0)$. This last equation gives the relation between the Bohmian trajectory $x(t)$ and the classical, Newtonian trajectory $\bar{x}(t)$.

It transpires from equation (58) that the sign of $\kappa$ plays a crucial role in determining the asymptotic features of the Bohmian trajectories. Let us consider this in more detail, in connection with the stability of the wave packets of stationary width, first for the measurement of the position of a free particle. If $\kappa>0$ (as implicitly assumed by Nassar and Miret-Artés [1,2]) the Bohmian trajectories tend to approach the classical one as $t \rightarrow \infty$. That is, the classical trajectory $\bar{x}(t)$ is an attractor of the Bohmian trajectories. On the contrary, if $\kappa<0$ the Bohmian trajectories deviate exponentially from the classical trajectory. This may suggest that $\kappa>0$ is the appropriate choice for modelling the position measurement. However, a different picture emerges if one considers the dependence on the sign of $\kappa$ of the wave packet dynamics, and in particular, of the time-evolution of the width $\delta$ of the probability density $\rho=|\psi|^{2}$. In this regard what really matters, is the behaviour of the probability $\operatorname{density~} \rho(x, t)=|\psi(x, t)|^{2}$, which tells us how probable it is to find the measured particle at different locations at a given time $t$. For $\kappa>0$ the 


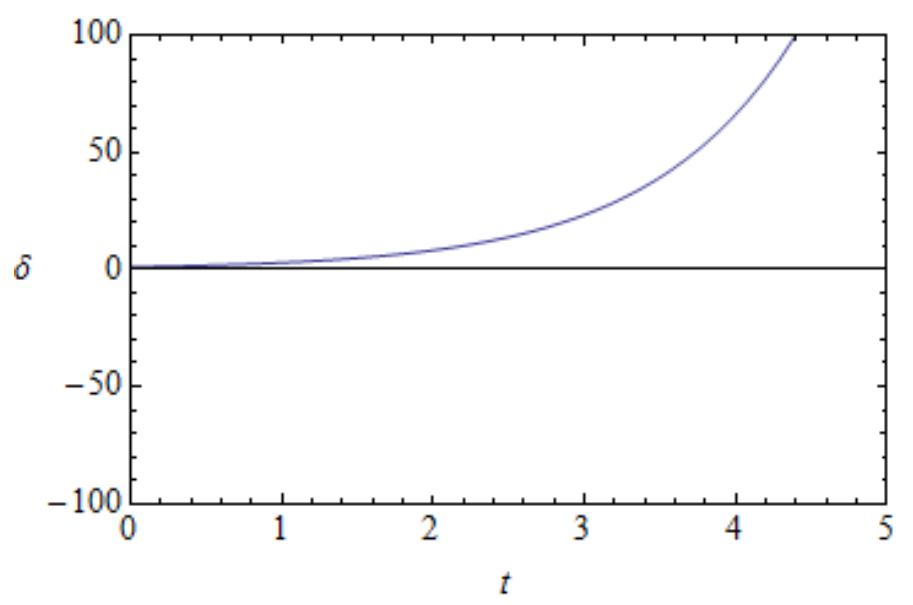

(a)Evolution of $\delta$ for an unbound classical orbit $\bar{x}(t)(50)$ for $\mu=2$ with $\delta(0)=1, \delta^{\prime}(0)=1$.

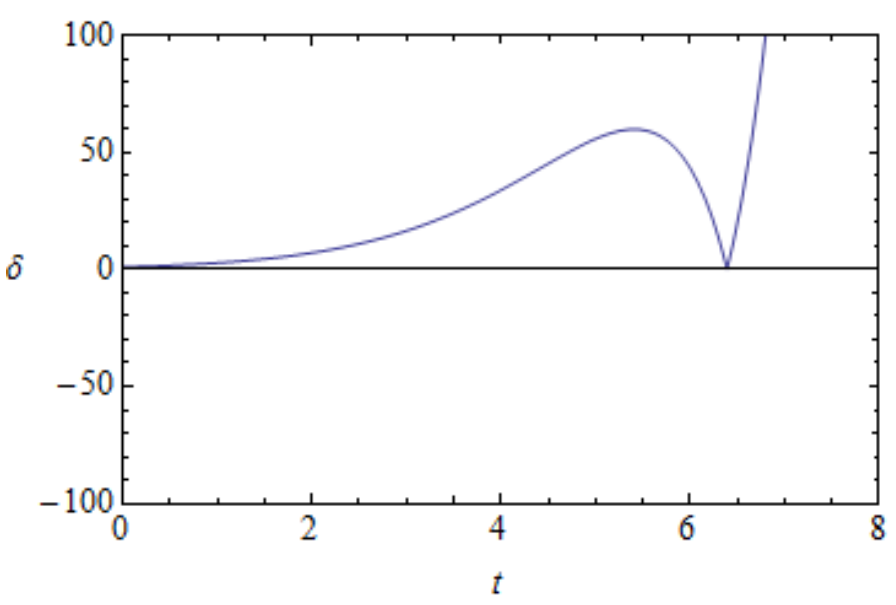

(b)Evolution of $\delta$ for a bound classical orbit $\bar{x}(t)(52)$ for $\mu=0.5$ with $\delta(0)=1, \delta^{\prime}(0)=1$.

FIG. 6: Time evolution of the wave packet width, $\delta(t)$, in a Morse potential for bound and unbound classical orbits $\bar{x}(t)$ of the wave packet center and different initial conditions $\delta(0)$ and $\delta^{\prime}(0)$. The relevant parameters are $\kappa=1, D=1, \alpha=0.1, x_{0}=0$, $t_{0}=2$.

stationary wave packet considered by NM becomes unstable. Time-dependent wave packets starting from generic initial conditions evolve away from this stationary wave packet. Consequently, for $\kappa>0$ this static wave packet does not constitute a physically acceptable description of a continuous position measurement process. Nor does the $\mathrm{NM}$ equation itself provide for $\kappa>0$ a sensible account of the position measurement, since time-dependent wave packet solutions tend to become more de-localized as time progresses. For $\kappa<0$ the stationary wave packet is stable. Time-dependent Gaussian wave packets with general initial conditions evolve towards the stationary wave packet, which is now an attractor of the wave packet dynamics. For $\kappa<0$ initially spread wave packets become more localized as time progresses. This implies that the probability density $\rho(x, t)$ of the particle undergoing continuous position measurement becomes more localized as $t \rightarrow \infty$. This indicates that $\kappa<0$ leads to a physically appealing description of the measurement process.

In the same vein we are going to assume $\kappa<0$ for the linear potential and that condition (32) holds for the quadratic potential, which leads to the stability of $\delta(t)$ in (39) and (43) respectively. We then have a "localized" wave packet around the classical trajectory, even if we loose the property of the Bohmian trajectories approaching the classical one.

The stability of the stationary solution and resulting localization of the wave packet as discussed above, can be understood from a careful analysis of equation (13), regarding it as a balance equation for the probability density $\rho(x, t)$. This balance equation only becomes a continuity equation for $\kappa=0$, where one recovers the continuity equation associated with the standard linear Schrödinger equation. In the general case $\kappa \neq 0$, equation $(13)$ has a source term

$$
R(x, t)=-2 \kappa[\ln \rho-\langle\ln \rho\rangle] \rho .
$$

In order to understand the effect of this source term that cannot be written as the divergence of a probability density current, it is instructive to consider the simplified equation

$$
\frac{\partial \rho}{\partial t}=-2 \kappa[\ln \rho-\langle\ln \rho\rangle] \rho
$$

where the probability density $\rho$ evolves under the sole effect of the source term. The solution to this equation is (see Appendix B for details)

$$
\rho(x, t)=\frac{\exp \left[e^{-2 \kappa t} \ln \rho(x, 0)\right]}{\int_{-\infty}^{+\infty} \exp \left[e^{-2 \kappa t} \ln \rho\left(x^{\prime}, 0\right)\right] d x^{\prime}} .
$$


It is interesting to note that it follows from expression (61) that the probability density $\rho(x, t)$ at time $t$ has the form of an escort distribution $[40,41]$ of the initial density $\rho(x, 0)$. In fact, by defining $q=\exp [-2 \kappa t]$ we have

$$
\rho(x, t)=\frac{\rho(x, 0)^{q}}{\int_{-\infty}^{+\infty} \rho\left(x^{\prime}, 0\right)^{q} d x^{\prime}} .
$$

For an initial Gaussian distribution $\rho(x, 0)=\frac{1}{\sqrt{2 \pi}} e^{-x^{2} / 2}$, equation (61) becomes

$$
\rho(x, t)=\frac{1}{\sqrt{2 \pi e^{2 \kappa t}}} \exp \left[-\frac{x^{2}}{2 e^{2 \kappa t}}\right]
$$

and equation (59) is then

$$
R(x, t)=-2 \kappa\left[\frac{1}{2}-\frac{x^{2}}{2 e^{2 \kappa t}}\right] \rho(x, t) .
$$

Figures $7(\mathrm{a})$ and $7(\mathrm{~b})$ show equation (63) for $\kappa=1$ and -1 respectively. Figure 8 shows plots for the source term equation (64) for $\kappa=1$ and -1 , and for different times. From Figure 7 one can see that for positive $\kappa$ the width of the Gaussian wave packet increases with time, whereas for negative $\kappa$ it decreases. The decrease in the dispersion would be consistent with the particle's position being measured, thus implying that $\kappa$ should be negative. In terms of the Bohmian approach, Figure 8 illustrates the reason for these different behaviours. For $\kappa>0$ the probability density of the Bohmian particle exhibits a sink (negative source) near the centre and a positive source further away, with the sink region spreading in time. When $\kappa<0$ the source is positive around the centre of the distribution, with the width of this region being reduced with time.

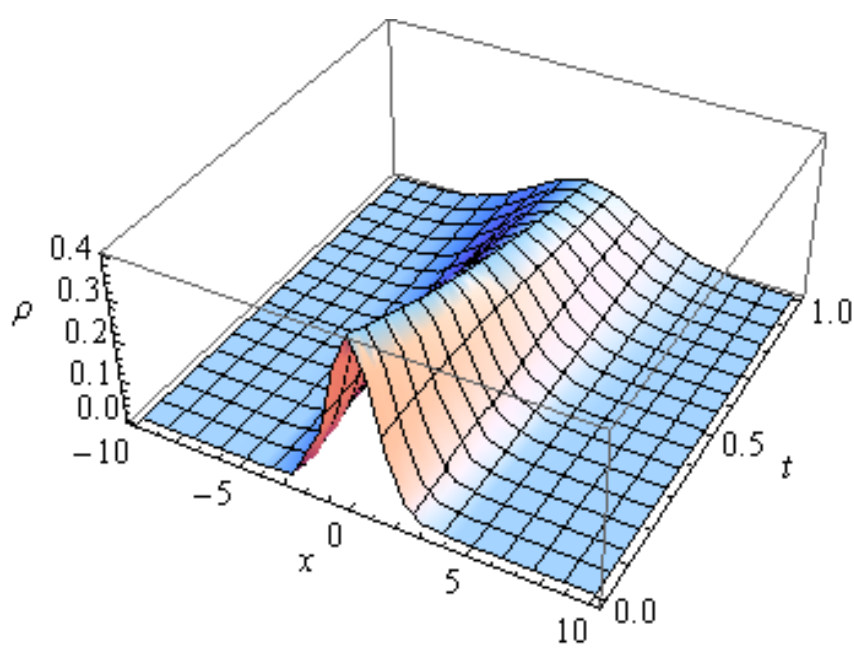

(a) $\kappa=1$.

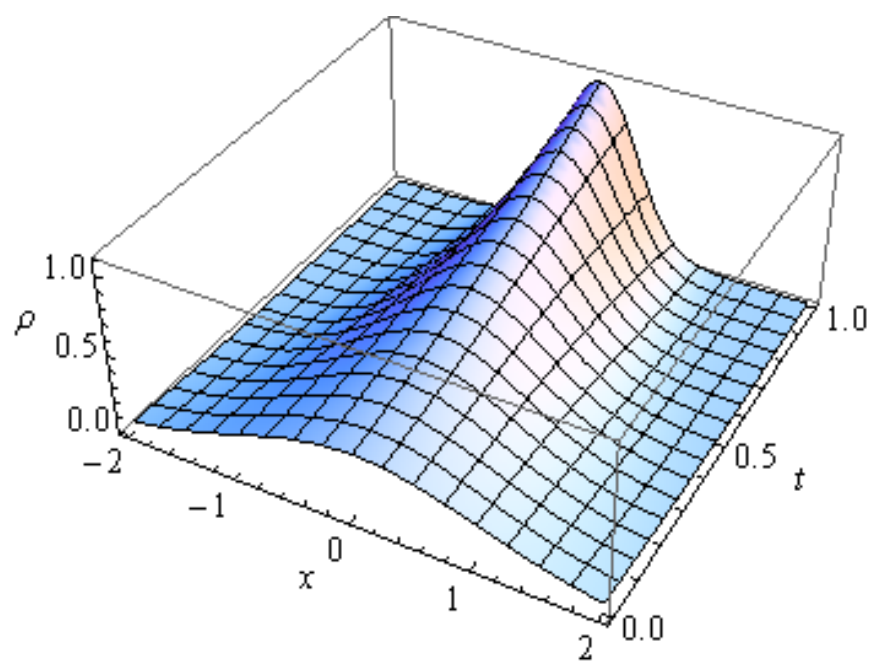

(b) $\kappa=-1$.

FIG. 7: Space-time behavior of the probability density $\rho(x, t)$ (eq. (63)) evolving under the effect of the source term (59) for positive and negative values of $\kappa$.

The overall evolution of the probability density $\rho(x, t)$ results from the competition of two processes. On the one hand we have the motion of the Bohmian particle giving rise to the probability density current $\rho u$, where $u$ is the Bohmian velocity field. On the other hand we have the sources and sinks of probability described by $R(x, t)$ in (59). When $\kappa>0$ the Bohmian trajectories converge to the classical one, and the current $\rho u$ tends to localize the probability density $\rho$. However, the source term $R(x, t)$ has the opposite effect on $\rho$ when condition (32) is not satisfied for the linear and quadratic potentials, and is then dominating. This explains the unstable character of the stationary solution discussed in Subsection III B.

When $\kappa<0$ the Bohmian trajectories exhibit an exponential deviation from the classical trajectory $\bar{x}$. This leads to a current $\rho u$ that tends to de-localize the probability density $\rho(x, t)$. However, in this case the source term $R(x, t)$ 


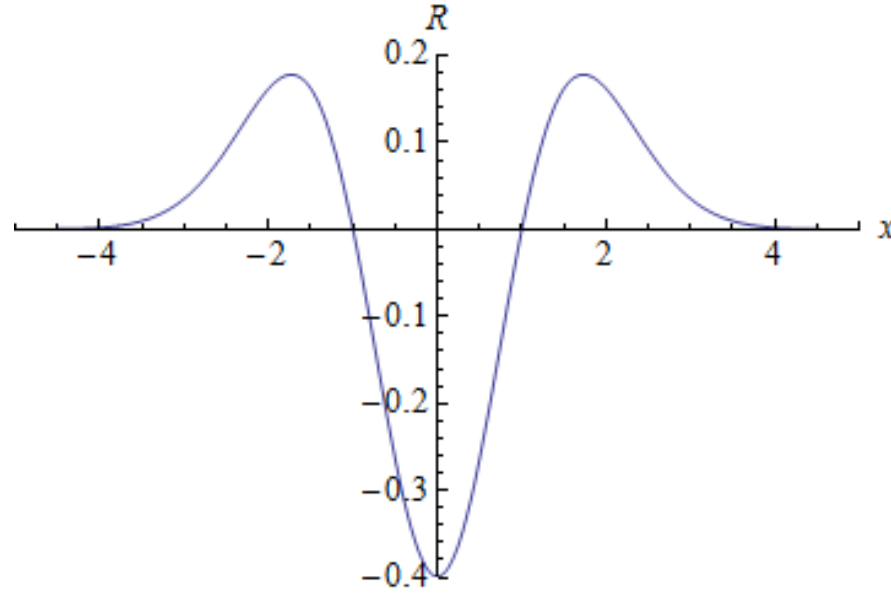

(a) $\kappa=1$ and $t=0$.

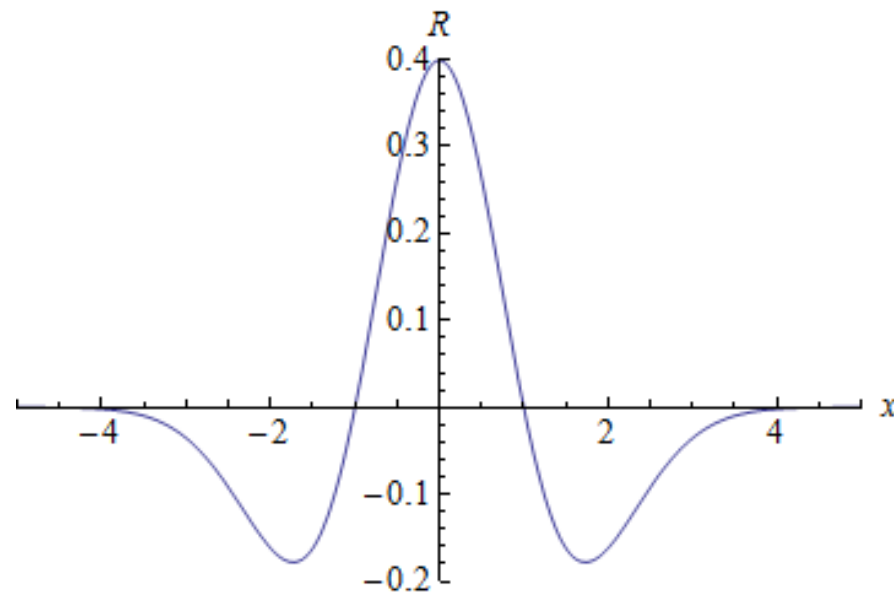

(c) $\kappa=-1$ and $t=0$.

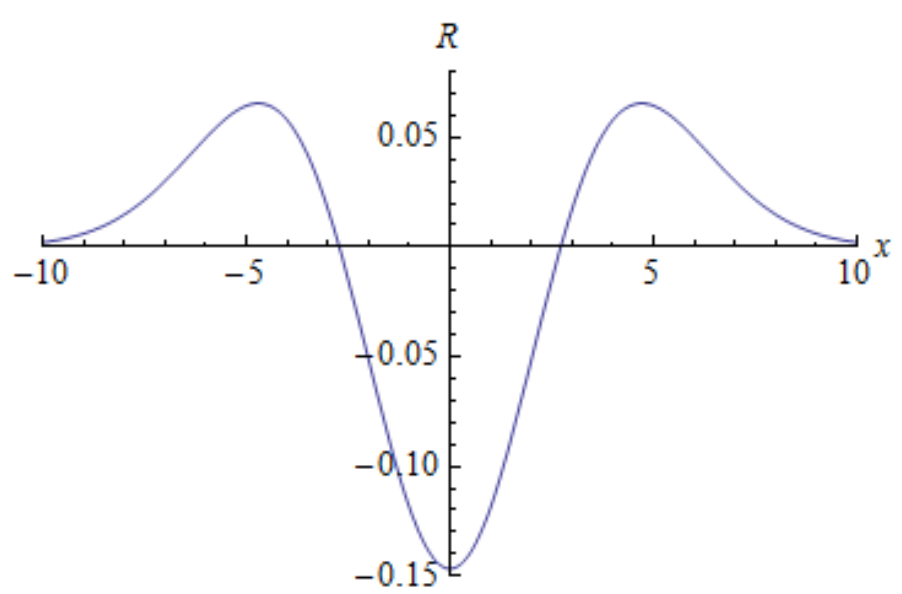

(b) $\kappa=1$ and $t=1$.

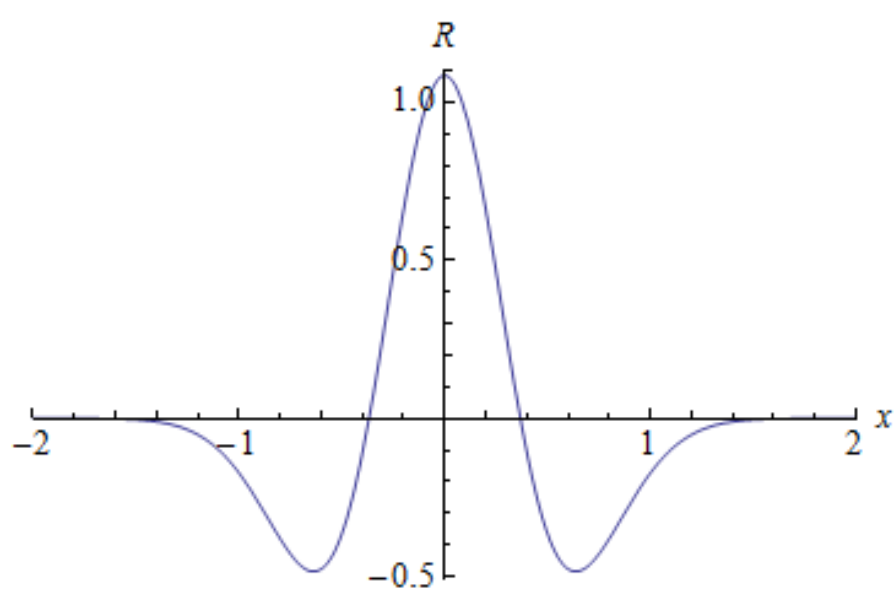

(d) $\kappa=-1$ and $t=1$.

FIG. 8: Plots of the source term (64) for different values of $\kappa$ and at different times.

(which is again the dominating term) has the opposite effect, resulting in a stable solution. For the free particle and linear potential, $\kappa<0$ leads to wave packet solutions that approach the stationary localized solution, whereas for the quadratic potential condition (32) has to be fulfilled. This behaviour is consistent with a continuous quantum position measurement process.

The effect of the source term $R(x, t)$ (when $\kappa<0$ ) on a free particle can be interpreted as describing the collapse of the particle's wave function due to it being measured. On the other hand, the deviation of the Bohmian trajectories from the classical path corresponds to the natural tendency of a free particle's evolving wave packet to spread.

It is worth mentioning that Mensky's path integral approach to continuous measurements, which was one of the heuristic motivations behind the NM equation [1], also points towards a negative value of the parameter $\kappa$. On the basis of this path integral approach, Mensky proposed a Schrödinger equation involving a non-Hermitian effective Hamiltonian,

$$
i \hbar \frac{\partial \psi(x, t)}{\partial t}=\left[H(x, t)-i \Gamma(x-\bar{x}(t))^{2}\right] \psi(x, t),
$$

with $\Gamma$ a positive real parameter with dimensions of energy [3]. The dynamical evolution determined by Mensky's equation (65) does not preserve the norm of the wave function, while the NM dynamics does. The first step to go 
from Mensky's to the NM dynamics is to modify (65) so that the norm is preserved, obtaining

$$
i \hbar \frac{\partial \psi(x, t)}{\partial t}=\left[H(x, t)-i \Gamma\left((x-\bar{x}(t))^{2}-\left\langle(x-\bar{x}(t))^{2}\right\rangle\right)\right] \psi(x, t) .
$$

Then, the basic procedure to establish a heuristic analogy between Mensky's equation and the NM equation is to identify, for a Gaussian wave packet having $|\psi|^{2} \propto \exp \left[-(x-\bar{x}(t))^{2} / 2 \delta^{2}\right]$, the term $i \Gamma\left((x-\bar{x}(t))^{2}-\left\langle(x-\bar{x}(t))^{2}\right\rangle\right)$ in Mensky's equation with the term $i \hbar \kappa\left[\ln |\psi(x, t)|^{2}-\left\langle\ln |\psi(x, t)|^{2}\right\rangle\right]$ in the NM equation. It is then clear that a positive $\Gamma$ leads to a negative $\kappa$. The above is just a heuristic argument, not a formal derivation. The results reported in this work do not depend on the analogy between Mensky's and the NM equation.

\section{EVOLUTION OF THE ENTROPY}

We now investigate the evolution of the entropy $S[\rho]$ of the particle's probability density $\rho$ satisfying the evolution equation (13) (which arises, in turn, from the non-linear Schrödinger equation (5)). Entropic measures constitute useful tools for characterizing both static and dynamic aspects of the degree of localization exhibited by quantum mechanical probability densities $[42,43]$. In particular, they provide a clear way of visualizing the time evolution of the localization [43].

Let us consider the entropy

$$
\mathcal{S}[\rho]=-\int \rho \ln \rho d x
$$

and its time derivative

$$
\frac{d \mathcal{S}}{d t}=-\int \frac{\partial \rho}{\partial t} \ln \rho d x
$$

where the preservation of normalization of $\rho$ was taken into account. Substituting equation (13) into (68), performing integration by parts and neglecting surface terms since we assume that $\rho \rightarrow 0$ fast enough as $|x| \rightarrow \infty$, we obtain

$$
\frac{d \mathcal{S}}{d t}=\left[\int \frac{\partial u}{\partial x} \rho d x\right]+2 \kappa \int \rho(\ln \rho-\langle\ln \rho\rangle)^{2} d x .
$$

Using the ansatz in equation (15) the entropy becomes

$$
S(\delta)=\ln \sqrt{2 \pi \delta(t)^{2}}+\frac{1}{2}
$$

and its derivative

$$
\frac{d \mathcal{S}}{d t}=\frac{\dot{\delta}}{\delta}-\kappa+2 \kappa \int \rho(\ln \rho-\langle\ln \rho\rangle)^{2} d x
$$

Figure 9 shows the entropy for the quadratic and the Morse potentials for specific solutions of $\delta(t)$ indicated in the caption.

It is plain from equations (69) and (71) that $\frac{d \mathcal{S}}{d t}$ does not have a definite sign. However, it can be clearly separated into two parts of different origin. This is related to the structure of the evolution equation $(13)$ for the density $\rho(x, t)$,

$$
\frac{\partial \rho}{\partial t}=\underbrace{-\frac{\partial}{\partial x}(\rho u)}_{\text {"local flux" term }}-\underbrace{2 \kappa[\ln \rho-\langle\ln \rho\rangle] \rho}_{\text {source term }} .
$$

We see that, as already mentioned, the evolution equation for $\rho$ is not a continuity equation. The normalization $\int \rho d x$ is conserved "globally", but the probability density is not conserved "locally" (its local change is not solely given by a flux). This manifests itself in the structure of $\frac{d \mathcal{S}}{d t}(71)$,

$$
\frac{d \mathcal{S}}{d t}=\underbrace{\frac{\dot{\delta}}{\delta}-\kappa}_{\text {"local" part }}+\underbrace{2 \kappa \int \rho(\ln \rho-\langle\ln \rho\rangle)^{2} d x}_{\text {"non-local" part }} .
$$




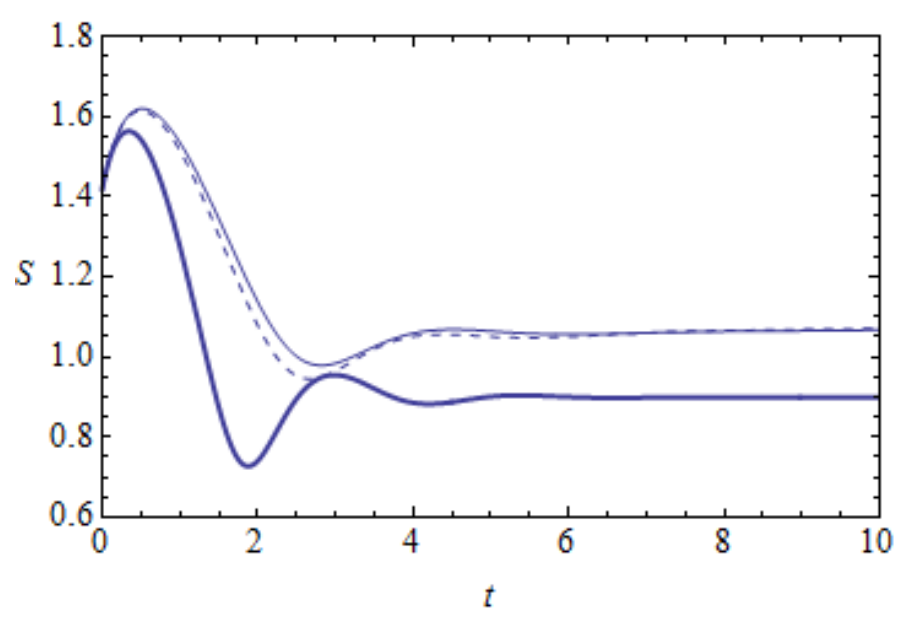

FIG. 9: Graph of the entropy (70) of the probability density $\rho$ corresponding to the solutions of $\delta(t)$ plotted in Figures $4(\mathrm{~b})$ (thick line), 5(b) (dashed line) and $5(\mathrm{~d})$.

We see that the "local" part does not have a definite sign. The "non-local" term, on the contrary, has a definite sign depending on the sign of $\kappa$.

The second, non-local term in the right hand side of equation (69) arises solely from the non-linear term in the NM-equation. That is, in the standard case of the linear Schrödinger equation this term would vanish. It is clear that in order for this term (which has a definite sign) to contribute to the localization effect of the NM dynamics (corresponding to a decreasing $S$ ) the parameter $\kappa$ has to be negative. The entropic analysis then supports our previous conclusion, based on the analysis of the stability conditions of the stationary wave packet solutions, that one has to have $\kappa<0$.

Notice that the instantaneous value of $d S / d t$ at a given instant of time is completely determined by the instantaneous wave function $\psi(x)$ at that particular time, since both $\rho$ and $u$ are determined by $\psi$, through equations (9-11) (here we dropped the time argument from $\psi(x)$ to emphasize that our present considerations refer to the wave function at a given time). It is interesting to compare the behaviors of the two terms in the right hand side of equation (69) under a scale transformation of the wave function that changes its spatial spread. To do that, let us consider the transformation,

$$
\psi(x) \rightarrow \tilde{\psi}(x)=\frac{1}{\sqrt{\lambda}} \psi\left(\frac{x}{\lambda}\right)
$$

where $\lambda>0$ is a scale parameter (one can regard the re-scaled $\tilde{\psi}$, for instance, as a possible initial condition for the NM evolution equation). It is clear that the above transformation preserves the normalization of the wave function. It also preserves the shape of the wave function: $\psi$ and $\tilde{\psi}$ have the same shape but, for $\lambda>1, \tilde{\psi}$ is more spread spatially than $\psi$. The transformation (74) of the wave function leads to the following transformations of the density $\rho$ and the velocity field $u$,

$$
\rho(x) \rightarrow \tilde{\rho}(x)=\frac{1}{\lambda} \rho\left(\frac{x}{\lambda}\right)
$$

and

$$
u(x) \rightarrow \tilde{u}(x)=\frac{1}{\lambda^{3 / 2}} u\left(\frac{x}{\lambda}\right)
$$

By recourse to the above relations we can evaluate the entropic time derivative $\frac{d S}{d t}[\tilde{\rho}]$ corresponding to the transformed wave function $\tilde{\psi}$. It can be verified after some calculations that,

$$
\frac{d S}{d t}[\tilde{\rho}]=\left[\int \frac{\partial \tilde{u}}{\partial x} \tilde{\rho} d x\right]+2 \kappa \int \tilde{\rho}(\ln \tilde{\rho}-\langle\ln \tilde{\rho}\rangle)^{2} d x
$$




$$
=\frac{1}{\lambda^{3 / 2}}\left[\int \frac{\partial u}{\partial x} \rho d x\right]+2 \kappa \int \rho(\ln \rho-\langle\ln \rho\rangle)^{2} d x .
$$

We see that the non-local term in $d S / d t$, arising from the source term in the evolution equation for the density $\rho$, is invariant under a scaling transformation (74). On the contrary, the local term in $d S / d t$ gets re-scaled, and tends to zero when $\lambda \rightarrow \infty$. This means that in the limit of large $\lambda$-values the second term in the expression for $d S / d t$ (see equation (77)) is dominant,

$$
\lim _{\lambda \rightarrow \infty} \frac{d S}{d t}[\tilde{\rho}]=2 \kappa \int \tilde{\rho}(\ln \tilde{\rho}-\langle\ln \tilde{\rho}\rangle)^{2} d x
$$

and thus the sign of $d S / d t$ is given by the sign of $\kappa$. One can formulate this result in a way akin to an $H$-theorem $[44,45]$ : For highly spread wave functions of any shape (that is, taking $\lambda$ in (74) large enough) the sign of $d S / d t$ is equal to the sign of $\kappa$. This, in turn, implies that $\kappa$ has to be negative for the non-linearity in the NM equation to give rise to a localization effect in the evolution of an initially highly spread wave function.

\section{CONCLUSIONS}

In the present contribution we studied a non-linear extension of the Schrödinger equation, which describes a quantum particle moving in a potential under the influence of a dissipative effect and evolving under a continuous position measurement process.

We obtained exact wave packet time-dependent solutions for the cases of constant, linear and quadratic potentials. We also developed a procedure yielding approximate wave packet solutions for more general cases, which we illustrated for the Morse potential.

On the basis of the above results we explored the stability properties of the stationary solutions (that is, wave packet solutions of constant width $\delta$ ) of the NM equation. We determined the constraints on the values of the parameters $\kappa$ and $\nu$ appearing in the NM equation under which the stationary solutions are attractors of the wave packet dynamics, and consequently are stable solutions. If these constraints are not satisfied, the NM dynamics exhibits physically undesirable features that renders it unacceptable as a description of a position measurement process.

We showed that for the free particle and the linear and quadratic potentials, and for appropriate values of $\kappa$ and $\nu$, the system relaxes to a state localized at each time $t$ around the corresponding point $\bar{x}(t)$ on the classical trajectory, with the width of the wave packet approaching a stationary value. An expression for the characteristic relaxation time was also obtained. The approximate wave packet solutions that we obtained for the Morse potential indicate that the width of the wave packet evolves towards a time-periodic attractor.

The dynamics corresponding to the linear and the quadratic potentials is very similar whether $\nu=0$ or $\nu \neq 0$, as long as the inequalities (32) required for the stability of the stationary solution are satisfied. Thus the additional dissipative friction term $W_{f}$ affects the dynamics mostly by changing $\delta_{s}$, which amounts to changing the resolution of the measurement. The dissipative dynamics due to the term $W_{f}$ and the effect of the continuous measurement described by $W_{c}$ both act in the same way, the two mechanism playing intertwining roles. In the cases of the free particle and the linear and quadratic potentials, the system relaxes to a wave packet with stationary width $\delta_{s}$ irrespective of the choice of the initial conditions for $\delta$.

When interpreting the NM dynamics from the Bohmian point of view it is essential to explicitly take into account that, even though the total probability $\int \rho(x, t) d x$ is conserved, the evolution equation for the probability density $\rho(x, t)$ is not a continuity equation. In fact, there are two contributions to the dynamics of $\rho(x, t)$. We have a probability density current $\rho u$ that originates on the Bohmian velocity field $u$, and we have a field of sources and sinks of probability, described by the logarithmic terms in the evolution equation (13) for $\rho(x, t)$. When the NM equation admits stable stationary solutions (that is, stable wave packet solutions with a stationary width $\delta_{s}$ ) the source field, which tends to localize the probability density, dominates over the probability density current, which has the opposite effect. 


\section{ACKNOWLEDGEMENTS}

C.Z. gratefully acknowledges the financial support of the University of Pretoria. Part of this research was conducted during an academic visit of C.Z. to the Universidad Nacional del Noroeste de la Prov. de Buenos Aires (UNNOBA) and the Universidad Nacional de La Plata. She wishes to express her gratitude for the kind hospitality of both institutions. A.R.P. acknowledges the support of the CEI of the Oviedo University through a grant BOPA n:97; $28 / 04 / 2014$

\section{Appendix A: Stability Conditions for the Stationary Wave Packets}

Now we are going to consider the stability conditions of the stationary solutions $\delta=\delta_{s}$ of the equation of motion,

$$
\ddot{\delta}=(2 \kappa-\nu) \dot{\delta}-\frac{\partial W}{\partial \delta},
$$

where

$$
W(\delta)=\frac{1}{2}\left(\kappa^{2}-\kappa \nu\right) \delta^{2}+\frac{\hbar^{2}}{8 m^{2} \delta^{2}}+\frac{1}{2 m} \delta^{2} V^{\prime \prime},
$$

with $V^{\prime \prime}$ constant. In this case the effective potential $W$ depends only on $\delta$ (that is, it is time-independent). We have

$$
W^{\prime}=\left(\kappa^{2}-\kappa \nu+\frac{V^{\prime \prime}}{m}\right) \delta-\frac{\hbar^{2}}{4 m^{2} \delta^{3}},
$$

and

$$
W^{\prime \prime}=\left(\kappa^{2}-\kappa \nu+\frac{V^{\prime \prime}}{m}\right)+\frac{3 \hbar^{2}}{4 m^{2} \delta^{4}} .
$$

In order for $\delta_{s}$ to be a stable stationary point of (A1) the drag force in the right hand side of (A1) has to be dissipative, and the potential $W(\delta)$ has to have a local minimum at $\delta_{s}$. A dissipative drag force requires $2 \kappa-\nu<0$. Therefore, in order to have stability we have the first condition,

$$
\kappa<\frac{\nu}{2}
$$

The condition for a stationary point, $W^{\prime}\left(\delta_{s}\right)=0$, yields,

$$
\kappa^{2}-\kappa \nu+\frac{V^{\prime \prime}}{m}=\frac{\hbar^{2}}{4 m^{2} \delta_{s}^{4}}>0 .
$$

Therefore $W$ has a stationary point $\delta_{s}$ iff

$$
\kappa^{2}-\kappa \nu+\frac{V^{\prime \prime}}{m}>0
$$

It follows from (A4) and (A6) that $W^{\prime}\left(\delta_{s}\right)=0$ implies $W^{\prime \prime}\left(\delta_{s}\right)>0$. That is, if $W$ has a stationary point at $\delta_{s}$ it is necessarily a minimum. Next, we determine the range of $\kappa$-values compatible with condition (A7). First notice that the roots of the left hand side of (A7) are,

$$
\frac{\nu}{2} \pm \sqrt{\frac{\nu^{2}}{4}-\frac{V^{\prime \prime}}{m}}
$$

Therefore, if $\frac{\nu^{2}}{4}-\frac{V^{\prime \prime}}{m}<0$ there are no real roots and condition (A7) holds for all $\kappa$-values. On the other hand, if $\frac{\nu^{2}}{4}-\frac{V^{\prime \prime}}{m} \geq 0$ we have two real roots. In this case (A7) holds either if $\kappa<\frac{\nu}{2}-\sqrt{\frac{\nu^{2}}{4}-\frac{V^{\prime \prime}}{m}}$ or if $\kappa>\frac{\nu}{2}+\sqrt{\frac{\nu^{2}}{4}-\frac{V^{\prime \prime}}{m}}$. The second case is incompatible with the constraint $\kappa<\nu / 2$. Consequently, in order to have a stable, stationary solution $\delta_{s}$ of $(\mathrm{A} 1)$ one has the requirements,

$$
\begin{array}{lll}
\kappa<\frac{\nu}{2} & \text { if } & \frac{\nu^{2}}{4}-\frac{V^{\prime \prime}}{m}<0 \\
\kappa<\frac{\nu}{2}-\sqrt{\frac{\nu^{2}}{4}-\frac{V^{\prime \prime}}{m}} & \text { if } & \frac{\nu^{2}}{4}-\frac{V^{\prime \prime}}{m} \geq 0 .
\end{array}
$$


Appendix B: Behavior of the probability density $\rho$ evolving solely under the effect of the source term

Here we are going to show that the general time-dependent solution $\rho(x, t)$ to the evolution equation

$$
\frac{\partial \rho}{\partial t}=-2 \kappa[\ln \rho-\langle\ln \rho\rangle] \rho,
$$

where the probability density evolves under the sole effect of the source term, is given by,

$$
\rho(x, t)=\frac{\exp \left[e^{-2 \kappa t} \ln \rho(x, 0)\right]}{\int_{-\infty}^{+\infty} \exp \left[e^{-2 \kappa t} \ln \rho\left(x^{\prime}, 0\right)\right] d x^{\prime}},
$$

where $\rho(x, 0)$ is the initial density at $t=0$. In the proof that follows it will be convenient to define,

$$
L(t)=\ln \left(\int_{-\infty}^{+\infty} \exp \left[e^{-2 \kappa t} \ln \rho\left(x^{\prime}, 0\right)\right] d x^{\prime}\right) .
$$

First of all, notice that the time-dependent probability density (B2) is properly normalized for all times $t$, and that by setting $t=0$ in (B2) one recovers the initial condition $\rho(x, 0)$. Now, evaluating the partial time derivative of $\rho(x, t)$ as given by the expression in the right hand side of (B2) one gets,

$$
\begin{aligned}
\frac{\partial}{\partial t} \rho(x, t) & =\frac{\partial}{\partial t}\left[\frac{\exp \left[e^{-2 \kappa t} \ln \rho(x, 0)\right]}{\int_{-\infty}^{+\infty} \exp \left[e^{-2 \kappa t} \ln \rho\left(x^{\prime}, 0\right)\right] d x^{\prime}}\right]=\frac{-2 \kappa e^{-2 \kappa t}(\ln \rho(x, 0)) \exp \left[e^{-2 \kappa t} \ln \rho(x, 0)\right]}{\int_{-\infty}^{+\infty} \exp \left[e^{-2 \kappa t} \ln \rho\left(x^{\prime}, 0\right)\right] d x^{\prime}} \\
& +\frac{2 \kappa e^{-2 \kappa t} \exp \left[e^{-2 \kappa t} \ln \rho(x, 0)\right] \int_{-\infty}^{+\infty}\left(\ln \rho\left(x^{\prime}, 0\right)\right) \exp \left[e^{-2 \kappa t} \ln \rho\left(x^{\prime}, 0\right)\right] d x^{\prime}}{\left(\int_{-\infty}^{+\infty} \exp \left[e^{-2 \kappa t} \ln \rho\left(x^{\prime}, 0\right)\right] d x^{\prime}\right)^{2}} \\
& =-2 \kappa e^{-2 \kappa t}(\ln \rho(x, 0)) \rho(x, t)+2 \kappa e^{-2 \kappa t} \rho(x, t) \frac{\int_{-\infty}^{+\infty}\left(\ln \rho\left(x^{\prime}, 0\right)\right) \exp \left[e^{-2 \kappa t} \ln \rho\left(x^{\prime}, 0\right)\right] d x^{\prime}}{\int_{-\infty}^{+\infty} \exp \left[e^{-2 \kappa t} \ln \rho\left(x^{\prime}, 0\right)\right] d x^{\prime}} \\
& =-2 \kappa\left(e^{-2 \kappa t} \ln \rho(x, 0)-\frac{\int_{-\infty}^{+\infty} e^{-2 \kappa t}\left(\ln \rho\left(x^{\prime}, 0\right)\right) \exp \left[e^{-2 \kappa t} \ln \rho\left(x^{\prime}, 0\right)\right] d x^{\prime}}{\int_{-\infty}}\right) \rho(x, t) \\
& =-2 \kappa\left(e^{-2 \kappa t} \ln \rho(x, 0)-L(t)-\int_{-\infty}^{+\infty}\left[e^{-2 \kappa t}\left(\ln \rho\left(x^{\prime}, 0\right)\right)-L(t)\right] \rho\left(x^{\prime}, t\right) d x^{\prime}\right) \rho(x, t) \\
& =-2 \kappa\left(\ln \rho(x, t)-\int_{-\infty}^{+\infty}\left(\ln \rho\left(x^{\prime}, t\right)\right) \rho\left(x^{\prime}, t\right) d x^{\prime}\right) \rho(x, t) \\
& =-2 \kappa(\ln \rho(x, t)-\langle\ln \rho(x, t)\rangle) \rho(x, t),
\end{aligned}
$$

which completes the proof that expression (B2) satisfies the differential equation (B1).

[1] A.B. Nassar, Ann. Phys. 331 (2013) 317.

[2] A.B. Nassar and S. Miret-Artés, Phys. Rev. Lett 111 (2013) 150401.

[3] M.B. Mensky, Continuous Quantum Measurement and Path Integrals, IOP Publishing, Bristol, 1993; Phys. Lett. A 231 (1997) 1.

[4] M.D. Kostin, J. Chem. Phys. 57 (1972) 3589.

[5] F.D. Nobre, M.A. Rego-Monteiro and C. Tsallis, Phys. Rev. Lett. 106 (2011) 140601.

[6] A.R. Plastino, A.M.C. Souza, F.D. Nobre, and C. Tsallis, Phys. Rev. A 90 (2014) 062134.

[7] A.M. Scarfone, Phys. Rev. E 71 (2005) 051103.

[8] T.D. Frank, Nonlinear Fokker-Planck Equations, Springer-Verlag, Berlin, 2005.

[9] A.T. Silva, E.K. Lenzi, L.R. Evangelista, M.K. Lenzi and L.R. da Silva, Physica A 375 (2007) 65.

[10] I.T. Pedron, R.S. Mendes, T.J. Buratta, L.C. Malacarne, and E.K. Lenzi, Phys. Rev. E 72 (2005) 031106.

[11] T. Yamano Cent. Eur. Journ. Phys. 11 (2013) 910.

[12] A.C. Scott, The Nonlinear Universe (Springer, Berlin, 2007).

[13] J. Diaz-Alonso and D. Rubiera-Garcia, Phys. Rev. D 82 (2010) 085024. 
[14] T. Yamano and O. Iguchi, EPL 83 (2008) 50007.

[15] J.S. Bell, Speakable and Unspeakable in Quantum Mechanics, 2nd Edition, Cambridge University Press, 2004.

[16] M. Jammer, Concepts of Mass in Contemporary Physics and Philosophy, Princeton University Press, 2000.

[17] P.R. Holland, The Quantum Theory of Motion: An Account of the de Broglie-Bohm Causal Interpretation of Quantum Mechanics, Cambridge University Press, 1995.

[18] A. Valentini, H. Westman, Proc. R. Soc. Lond. Ser. A 461 (2005) 253.

[19] G. Naaman-Marom, N. Erez, L. Vaidman, Ann. Phys. 327 (2012) 2522.

[20] G.C. Ghirardi, A. Rimini, and T. Weber, Phys. Rev. D 34 (1986) 470.

[21] A. Caticha, J. Phys. A: Math. Theor. 44 (2011) 225303.

[22] B.R. Frieden and B.H. Soffer, Phys. Rev. E 52 (1995) 2274.

[23] M. Tegmark, arXiv:1401.1219v2.

[24] F. Pennini, A.R. Plastino and A. Plastino, Physica A 403 (2014) 195.

[25] A.B. Nassar and S. Miret-Artés, Ann. Phys. 348 (2014) 223.

[26] P. Holland, Ann. Phys. 315 (2005) 505.

[27] M. Tegmark, J.A. Wheeler, Sci. Am., Feb. 2001, p.72.

[28] P. Peter, E.J.C. Pinho, N. Pinto-Neto, Phys. Rev. D 75 (2007) 023516.

[29] N. Pinto-Neto, F.T. Falciano, R. Pereira, E.S. Santini, Phys. Rev. D 86 (2012) 063504.

[30] N. Pinto-Neto, G. Santos, W. Struyve, Phys. Rev. D 85 (2012) 083506.

[31] P.S. Letelier, J.P.M. Pitelli, Phys. Rev. D 82 (2010) 104046.

[32] C. Efthymiopoulos, C. Kalapotharakos, G. Contopoulos, Phys. Rev. E 79 (2009) 036203.

[33] A. Ramsak, EPL 96 (2011) 40004.

[34] A.F. Bennett, J. Phys. A: Math. Theor. 43 (2010) 195304.

[35] A.R. Plastino, M. Casas, A. Plastino Phys. Lett. A 281 (2001) 297.

[36] A. Valentini, J. Phys. A: Math. Theor. 40 (2007) 3285.

[37] A. Valentini, Phys. Rev. D 82 (2010) 063513.

[38] M.C. Gutzwiller, 1990 Chaos in Classical and Quantum Mechanics (Springer-Verlag New York).

[39] W.C. DeMarcus, Am. J. Phys. 46 (1978) 733.

[40] C. Beck and F. Schlogl, Thermodynamics of Chaotic Systems, Cambridge University Press, 1993.

[41] C. Tsallis, A.R. Plastino, and R.F. Alvarez-Estrada, J. Math. Phys. A 50 (2009) 043303.

[42] P.A. Bouvrie, A.P. Majtey, A.R. Plastino, P. Sanchez-Moreno, and J.S. Dehesa, Eur. Phys. J. D 66 (2012) 15.

[43] F.A. Cuevas, S. Curilef, and A.R. Plastino, Ann. Phys. 326 (2011) 2834.

[44] H.D. Zeh, The Physical Basis of the Direction of Time, Springer-Verlag, New York, 2007.

[45] J.A.S. Lima, R. Silva, and A.R. Plastino, Phys. Rev. Lett. 86 (2001) 2938. 